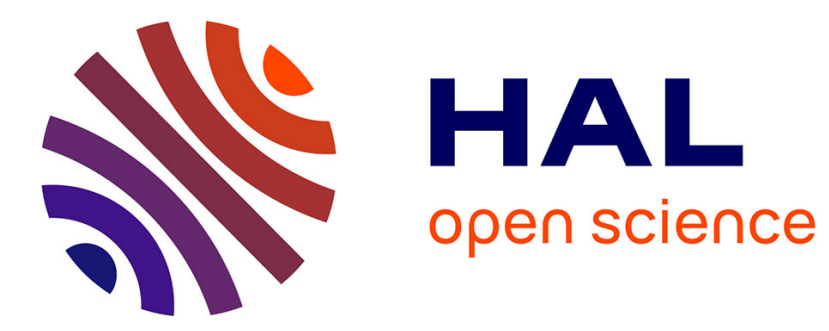

\title{
Stabilization of Persistently Excited Linear Systems by Delayed Feedback Laws
}

\author{
Guilherme Mazanti
}

\section{To cite this version:}

Guilherme Mazanti. Stabilization of Persistently Excited Linear Systems by Delayed Feedback Laws. 2013. hal-00850971v1

\section{HAL Id: hal-00850971 \\ https://hal.science/hal-00850971v1}

Preprint submitted on 10 Aug 2013 (v1), last revised 27 Mar 2014 (v2)

HAL is a multi-disciplinary open access archive for the deposit and dissemination of scientific research documents, whether they are published or not. The documents may come from teaching and research institutions in France or abroad, or from public or private research centers.
L'archive ouverte pluridisciplinaire HAL, est destinée au dépôt et à la diffusion de documents scientifiques de niveau recherche, publiés ou non, émanant des établissements d'enseignement et de recherche français ou étrangers, des laboratoires publics ou privés. 


\title{
Stabilization of Persistently Excited Linear Systems by Delayed Feedback Laws
}

\author{
Guilherme Mazanti
}

August 9, 2013

\begin{abstract}
This paper considers the stabilization to the origin of a persistently excited linear system by means of a linear state feedback, where we suppose that the feedback law is not applied instantaneously, but after a certain positive delay (not necessarily constant). The main result is that, under certain spectral hypotheses on the linear system, stabilization by means of a linear delayed feedback is indeed possible, generalizing a previous result already known for non-delayed feedback laws.
\end{abstract}

\section{Introduction}

Consider a control system of the form

$$
\dot{x}(t)=A x(t)+\alpha(t) B u(t), \quad x(t) \in \mathbb{R}^{d}, u(t) \in \mathbb{R}^{m}, \alpha \in \mathcal{G},
$$

where $x$ is the state variable, $u$ is a control input, $A$ and $B$ are matrices of appropriate dimensions, and $\alpha$ belongs to a certain class $\mathcal{G}$ of measurable scalar signals $\alpha: \mathbb{R}_{+} \rightarrow[0,1]$. This corresponds to the introduction on the linear control system $\dot{x}=A x+B u$ of a certain signal $\alpha$ that determines when and how much the control $u$ is active. Note that, when $\alpha$ takes its values on $\{0,1\},(1.1)$ is actually a switched system between the dynamics of the uncontrolled system $\dot{x}=A x$ and the controlled one $\dot{x}=A x+B u$.

Several different phenomena may be modeled by signal $\alpha$ in (1.1), such as a failure in the transmission of the control $u$ to the plant, a time-varying parameter affecting the control efficiency, or the allocation of control resources, among other possible phenomena. We are interested in general on robust control techniques of (1.1) with respect to $\alpha$ : we suppose that $\alpha$ is not precisely known and we wish our control strategy for (1.1) to be chosen independently of $\alpha$ and to be valid for any signal $\alpha$ in a certain class $\mathcal{G}$.

The problem of controlling (1.1) by a suitable choice of $u$ is obviously not interesting when $\alpha \equiv 0$, or when $\alpha$ is zero for a large amount of time, since in this case the control $u$ has a very limited effect on (1.1). The class $\mathcal{G}$ should thus ensure that the control input has a sufficient amount of action on the system. Among the possible choices for $\mathcal{G}$, the class of $(T, \mu)$-persistently exciting signals has attracted much interest recently (see, for instance, [7$10,13,16,20,22]$, and also [17] for a similar condition) and, for $T \geq \mu>0$, it consists on the signals $\alpha \in L^{\infty}\left(\mathbb{R}_{+},[0,1]\right)$ such that, for every $t \in \mathbb{R}_{+}$,

$$
\int_{t}^{t+T} \alpha(s) d s \geq \mu .
$$


The class of these signals $\alpha$ is noted $\mathcal{G}(T, \mu)$.

The condition of persistence of excitation (1.2) arises naturally in identification and adaptive control problems (see, e.g., $[1-3,6,18])$. In this context, we are led to study systems of the kind $\dot{x}=-P(t) x, x \in \mathbb{R}^{d}$, where $P(t)$ is a symmetric non-negative definite matrix for every $t$. If $P$ is bounded and has bounded derivative, it has been shown in [22] that the persistence of excitation of $P$, in the sense that $\alpha(t)=\xi^{\mathrm{T}} P(t) \xi$ is $(T, \mu)$-persistently exciting for all unitary vectors $\xi \in$ $\mathbb{R}^{d}$ and for certain constants $T \geq \mu>0$ independent of $\xi$, is a necessary and sufficient condition for the global exponential stability of $\dot{x}=-P(t) x$. This is what motivates the assumption that $\alpha$ is persistently exciting in (1.1). Further examples of systems similar to (1.1) where the persistent excitation condition appears are given in $[7,9,16]$, where the motivation for the use of persistently exciting signals is also more deeply discussed.

We consider the problem of stabilization of system (1.1) to the origin by means of a linear state feedback $u=-K x$, where we require the choice of the gain matrix $K$ not to depend on a particular signal $\alpha$ but instead on the class $\mathcal{G}(T, \mu)$. In many practical situations, this feedback cannot be done instantaneously, for a certain state $x(t)$ may not be available for measure before a certain delay $\tau$ has elapsed, and so the state measured in time $t$ is actually $x(t-\tau(t))$. This paper considers the problem of stabilization of (1.1) by a delayed feedback $u(t)=-K x(t-\tau(t))$, where the delay $\tau(t)$ may depend on $t$, and the closed-loop system becomes

$$
\begin{aligned}
& \dot{x}(t)=A x(t)-\alpha(t) B K x(t-\tau(t)), \\
& \alpha \in \mathcal{G}(T, \mu), \tau \in L^{\infty}\left(\mathbb{R}_{+}, \mathcal{T}\right)
\end{aligned}
$$

where $\mathcal{T} \subset \mathbb{R}_{+}$is the set where the delay $\tau$ takes its values. The goal of this paper is to present a stabilization result for system (1.3), showing that, under certain hypotheses on $A$ and $B$, given $T \geq \mu>0$ and $\tau_{0} \geq 0$, there exist a neighborhood $\mathcal{T}$ of $\tau_{0}$ in $\mathbb{R}_{+}$and $K \in \mathcal{M}_{m, d}(\mathbb{R})$ such that, for any $\alpha \in \mathcal{G}(T, \mu)$ and any delay function $\tau \in L^{\infty}\left(\mathbb{R}_{+}, \mathcal{T}\right)$, system (1.3) is exponentially stable, uniformly with respect to $\alpha$ and $\tau$. This generalizes [10, Theorem 3.2], where the same result is given in the case of the non-delayed feedback $u(t)=-K x(t)$, corresponding thus to $\mathcal{T}=\{0\}$.

Let us comment briefly on the technique used in [10] to prove this result in the non-delayed case. The main problem when dealing with the class $\mathcal{G}(T, \mu)$ is that a signal $\alpha \in \mathcal{G}(T, \mu)$ may be zero on certain time intervals, and so the system follows its uncontrolled dynamics $\dot{x}=A x$. On the other hand, for every $\rho>0$, it is known by a result from [11] that one can choose a linear feedback $u(t)=-K x(t)$ that stabilizes (1.1) uniformly with respect to $\alpha \in$ $L^{\infty}\left(\mathbb{R}_{+},[\rho, 1]\right)$. The main idea in [10] is to perform a change of variables corresponding to a time contraction by a factor $v>0$, which transforms a $(T, \mu)$-signal $\alpha$ into a $(T / v, \mu / v)$-signal $\alpha_{v}$ with $\alpha_{v}(t)=\alpha(v t)$. It is possible to show that the family $\left(\alpha_{v}\right)_{v>0}$ admits a weak- $\star$ convergent subsequence $\left(\alpha_{v_{n}}\right)_{n \in \mathbb{N}^{*}}$ in $L^{\infty}\left(\mathbb{R}_{+},[0,1]\right)$ with $v_{n} \rightarrow+\infty$ and that any weak- $\star$ subsequential limit $\alpha_{\star}$ of $\left(\alpha_{v}\right)_{v>0}$ as $v \rightarrow+\infty$ satisfies $\alpha_{\star}(t) \geq \mu / T$ almost everywhere. The idea is thus to study a certain limit system obtained as $v \rightarrow+\infty$, for which stabilization can be obtained using the result from [11] mentioned above. It can then be shown by a limit procedure that the same feedback gain $K$ also stabilizes a time-contracted system for a certain $v>0$ large enough, and one may finally adapt such a feedback gain $K$ in order to obtain a stabilizer for the original system.

This time-contraction technique used in [10] is well-adapted to deal with delays in the feedback, since a delay $\tau(t)$ in the original system will correspond to a delay $\frac{\tau(v t)}{v}$ in the timecontracted system. We may thus expect to obtain a non-delayed limit system as $v \rightarrow+\infty$ similar to the one obtained in [10] and to conclude the stabilizability of the original system by a similar argument. This intuition is actually true, as proved in Theorem 2.5 below, where we 
prove our stabilizability result by following the same time-contraction argument of the proof of [10, Theorem 3.2].

In their article [10], the authors first prove their stabilization result in the particular case where the dynamics are given by the Jordan block $J_{d}$ (see (3.1) below), since it is a representative example containing most of the difficulties of the proof of the general case. We also treat the case of the Jordan block separately in this article (see Theorem 3.1), but in this particular case we have a stronger result, showing that stabilizability is possible for any bounded interval $\mathcal{T} \subset \mathbb{R}_{+}$where the delay $\tau \in L^{\infty}\left(\mathbb{R}_{+}, \mathcal{T}\right)$ may take its values, whereas in the general case we may only guarantee stabilizability for delays $\tau$ which are perturbations around a certain constant prescribed value $\tau_{0}$. This difference between the statements of our result in the general case and in the particular case of the Jordan block is more deeply discussed in Section 5.

The plan of the paper is the following. In Section 2, we present the notations and definitions used throughout this paper and recall the previous result of [10]. We then proceed to prove, in Section 3, the main theorem of this paper in the particular case of the Jordan block, which allows us to highlight the main ideas of the proof in a setting where the notations are much clearer than in the general case, and also leads to a stronger result than in the general case. The proof of our main theorem is presented in Section 4, and Section 5 discusses the results we obtained, and specially the difference in the statements of Theorems 3.1 and 2.5. The proofs of some technical lemmas used in this paper are given in the Appendices A and B.

\section{Notations, Definitions and Previous Results}

In this paper, $\mathcal{M}_{d, m}(\mathbb{R})$ denotes the set of $d \times m$ matrices with real coefficients, which is denoted simply by $\mathcal{M}_{d}(\mathbb{R})$ when $d=m$. As usual, we identify column matrices in $\mathcal{M}_{d, 1}(\mathbb{R})$ with vectors in $\mathbb{R}^{d}$. The identity matrix in $\mathcal{M}_{d}(\mathbb{R})$ is denoted by $\operatorname{Id}_{d}$ and $0_{d \times m} \in \mathcal{M}_{d, m}(\mathbb{R})$ denotes the matrix whose entries are all zero, the dimensions being possibly omitted if they are implicit. The block-diagonal matrix whose diagonal blocks are the square matrices $a_{1}, \ldots, a_{d}$ is denoted by $\operatorname{diag}\left(a_{1}, \ldots, a_{d}\right)$. The notation $\|x\|$ indicates both the Euclidean norm of a vector $x \in \mathbb{R}^{d}$ and the associated matrix norm. The real and imaginary parts of a complex number $z$ are denoted by $\mathfrak{R}(z)$ and $\mathfrak{I}(z)$ respectively. The sets $\mathbb{R}_{+}$and $\mathbb{N}^{*}$ denote, respectively, the sets of the nonnegative real numbers $\mathbb{R}_{+}=[0,+\infty)$ and the positive integers $\mathbb{N}^{*}=\{1,2,3,4, \ldots\}$. For two topological spaces $X$ and $Y$, we denote by $\mathrm{C}^{0}(X, Y)$ the set of all continuous functions from $X$ to $Y$.

Throughout this paper, we consider the system

$$
\dot{x}(t)=A x(t)+\alpha(t) B u(t), \quad x(t) \in \mathbb{R}^{d}, u(t) \in \mathbb{R}^{m}, \alpha \in \mathcal{G}(T, \mu),
$$

where $A \in \mathcal{M}_{d}(\mathbb{R}), B \in \mathcal{M}_{d, m}(\mathbb{R})$, and we take persistently exciting signals $\alpha$ in the class $\mathcal{G}(T, \mu)$ defined as follows.

Definition 2.1. Let $T, \mu$ be two positive constants with $T \geq \mu$. We say that a measurable function $\alpha: \mathbb{R}_{+} \rightarrow[0,1]$ is a $(T, \mu)$-signal if, for every $t \in \mathbb{R}_{+}$, one has

$$
\int_{t}^{t+T} \alpha(s) d s \geq \mu
$$

The set of $(T, \mu)$-signals is denoted by $\mathcal{G}(T, \mu)$. System (2.1) with $\alpha \in \mathcal{G}(T, \mu)$ is called a persistently excited system (PE system for short). 
We shall consider the problem of stabilization of system (2.1) by means of a delayed linear state feedback $u(t)=-K x(t-\tau(t))$, where the delay $\tau$ is a function in $L^{\infty}\left(\mathbb{R}_{+}, \mathcal{T}\right)$ for a certain bounded set $\mathcal{T} \subset \mathbb{R}_{+}$and $K \in \mathcal{M}_{m, d}(\mathbb{R})$. With this feedback, system (2.1) takes the form

$$
\begin{aligned}
& \dot{x}(t)=A x(t)-\alpha(t) B K x(t-\tau(t)), \\
& \alpha \in \mathcal{G}(T, \mu), \tau \in L^{\infty}\left(\mathbb{R}_{+}, \mathcal{T}\right) .
\end{aligned}
$$

Note that, for $T \geq \mu>0$ and $\mathcal{T} \subset \mathbb{R}_{+}$bounded, for every $\alpha \in L^{\infty}\left(\mathbb{R}_{+},[0,1]\right)$ and every $\tau \in$ $L^{\infty}\left(\mathbb{R}_{+}, \mathcal{T}\right),(2.2)$ satisfies the Carathéodory conditions for delayed equations (see, for instance, [12, Section 2.6 and Theorem 6.1.1]), and so, noting $r=\sup \mathcal{T}$, for any given initial condition $x_{0} \in \mathrm{C}^{0}\left([-r, 0], \mathbb{R}^{d}\right),(2.2)$ admits a unique continuous solution $x$ defined on $[-r,+\infty)$, which is absolutely continuous on $\mathbb{R}_{+}$, coincides with $x_{0}$ on $[-r, 0]$, and satisfies $\dot{x}(t)=A x(t)-$ $\alpha(t) B K x(t-\tau(t))$ for almost every $t \in \mathbb{R}_{+}$. In order to make explicit the dependence of the solution $x$ on $\tau, x_{0}, \alpha$ and $K$, we denote $x(t)=x\left(t ; \tau, x_{0}, \alpha, K\right)$.

In the context of delayed systems, stability is defined in terms of the uniform norm of the initial condition (see, for instance, [12, Chapter 5]), which motivates the following definition.

Definition 2.2. Let $T \geq \mu>0$ and $\mathcal{T}$ be a bounded subset of $\mathbb{R}_{+}$, and denote $r=\sup \mathcal{T}$. We say that $K \in \mathcal{M}_{m, d}(\mathbb{R})$ is a $(T, \mu, \mathcal{T})$-stabilizer for (2.2) if there exist constants $C \geq 1$ and $\gamma>0$ such that, for every $\alpha \in \mathcal{G}(T, \mu)$, every $\tau \in L^{\infty}\left(\mathbb{R}_{+}, \mathcal{T}\right)$, and every initial condition $x_{0} \in \mathrm{C}^{0}\left([-r, 0], \mathbb{R}^{d}\right)$, the solution $x\left(t ; \tau, x_{0}, \alpha, K\right)$ of (2.2) satisfies

$$
\left\|x\left(t ; \tau, x_{0}, \alpha, K\right)\right\| \leq C e^{-\gamma t} \sup _{s \in[-r, 0]}\left\|x_{0}(s)\right\|, \quad \forall t \geq 0 .
$$

Remark 2.3. If $K$ is a $(T, \mu, \mathcal{T})$-stabilizer for (2.2), then, for every constant $\alpha_{\star} \in[\mu / T, 1]$ and every constant delay $\tau_{\star} \in \mathcal{T}$, the linear delayed system

$$
\dot{x}(t)=A x(t)-\alpha_{\star} B K x\left(t-\tau_{\star}\right)
$$

is exponentially stable. This is an important remark, since the stability and stabilization of systems with a constant delay of the form (2.3) can be more easily studied (see, for instance, $[21,23])$, giving rise to necessary conditions for $K$ to be a $(T, \mu, \mathcal{T})$-stabilizer. We shall use this approach later in Example 5.1.

Let us recall that a pair of matrices $(A, B) \in \mathcal{M}_{d}(\mathbb{R}) \times \mathcal{M}_{d, m}(\mathbb{R})$ is said to be stabilizable if there exists a matrix $K \in \mathcal{M}_{m, d}(\mathbb{R})$ such that $A-B K$ is Hurwitz. This is equivalent to saying that there exists an invertible matrix $P \in \mathcal{M}_{d}(\mathbb{R})$ such that

$$
P A P^{-1}=\left(\begin{array}{cc}
A_{1} & A_{3} \\
0 & A_{2}
\end{array}\right), \quad P B=\left(\begin{array}{c}
B_{1} \\
0
\end{array}\right),
$$

where $A_{2}$ is Hurwitz and $\left(A_{1}, B_{1}\right)$ is controllable. Stabilizability of a pair $(A, B)$ means that the linear control system $\dot{x}=A x+B u$ admits a linear state feedback $u=-K x$ such that the closedloop system $\dot{x}=(A-B K) x$ is exponentially stable, and thus, in order to achieve the required stabilizability property for system (2.2), the stabilizability of $(A, B)$ is a necessary condition when $0 \in \mathcal{T}$. This is what motivates us to consider only stabilizable pairs $(A, B)$ in what follows.

The stabilizability of (2.2) by means of a non-delayed feedback law has been studied in [10] in the case of a single-input system, i.e., when $m=1$, and it has been generalized to the multiinput case in [9]. In terms of Definition 2.2, this result can be stated as follows. 
Theorem 2.4. Let $(A, B) \in \mathcal{M}_{d}(\mathbb{R}) \times \mathcal{M}_{d, m}(\mathbb{R})$ be a stabilizable pair and assume that the eigenvalues of $A$ have non-positive real part. Then, for every $T \geq \mu>0$, there exists a $(T, \mu,\{0\})$ stabilizer for (2.2).

The hypothesis that the eigenvalues of $A$ have non-positive real part may seem restrictive, but it was shown in [10] that Theorem 2.4 is not true for certain stabilizable pairs $(A, B)$ and certain values of $T, \mu$ when $A$ admits an eigenvalue with positive real part. This is actually an effect of the signal $\alpha$ in the dynamics of the system; note that, when $\alpha(t) \in\{0,1\}$, the closedloop system actually switches between the dynamics given by $\dot{x}=A x$ and $\dot{x}=(A-B K) x$, and the phenomena related to this switch, such as the overshooting phenomenon, may lead to the non-stabilizability of the switched system when $A$ has an eigenvalue with positive real part, as detailed in [10]. For more general information on the behavior of switched systems, we refer to $[4,5,14,15,19,25]$.

The main result of this paper is the following generalization of Theorem 2.4.

Theorem 2.5. Let $(A, B) \in \mathcal{M}_{d}(\mathbb{R}) \times \mathcal{M}_{d, m}(\mathbb{R})$ be a stabilizable pair and assume that the eigenvalues of $A$ have non-positive real part. Then, for every $T \geq \mu>0$ and every $\tau_{0} \geq 0$, there exists a neighborhood $\mathcal{T}$ of $\tau_{0}$ in $\mathbb{R}_{+}$and a $(T, \mu, \mathcal{T})$-stabilizer for (2.2).

We prove this theorem here by generalizing the proof given in [10] in the non-delayed case. The main point is that the time-contraction argument given in [10], when applied to a delayed system, reduces the effects of the delay in the system, in such a way that the limit system obtained by making the time-contraction parameter tend to infinity is essentially the same in the delayed and the non-delayed cases. In order to highlight these main ideas, we first consider a particular case of Theorem 2.5.

\section{The $d$-Integrator}

Before turning to the proof of Theorem 2.5, let us first consider the particular case where the dynamics of the system are given by the $d$-integrator, defined by the Jordan block

$$
J_{d}=\left(\begin{array}{ccccccc}
0 & 1 & 0 & 0 & \cdots & 0 & 0 \\
0 & 0 & 1 & 0 & \cdots & 0 & 0 \\
0 & 0 & 0 & 1 & \cdots & 0 & 0 \\
0 & 0 & 0 & 0 & \cdots & 0 & 0 \\
\vdots & \vdots & \vdots & \vdots & \ddots & \vdots & \vdots \\
0 & 0 & 0 & 0 & \cdots & 0 & 1 \\
0 & 0 & 0 & 0 & \cdots & 0 & 0
\end{array}\right)
$$

and by taking $m=1$ and $B=\left(\begin{array}{llll}0 & \cdots & 0 & 1\end{array}\right)^{\mathrm{T}} \in \mathcal{M}_{d, 1}(\mathbb{R})$. This particular case will allow us to highlight the main ideas of the proof of Theorem 2.5, since it contains most of the difficulties of the general case. Furthermore, we can give in this case a stronger result, showing the existence of a $(T, \mu, \mathcal{T})$-stabilizer for any bounded interval $\mathcal{T} \subset \mathbb{R}_{+}$, and not only for perturbations around a certain value as in the general case of Theorem 2.5 .

Theorem 3.1. Let $A=J_{d}, B=\left(\begin{array}{llll}0 & \cdots & 0 & 1\end{array}\right)^{\mathrm{T}} \in \mathbb{R}^{d}$, and let $T \geq \mu>0$ and $r>0$ be given. Then there exists $a(T, \mu,[0, r])$-stabilizer $K \in \mathcal{M}_{1, d}(\mathbb{R})$ for (2.2). 
Proof. The proof follows the same idea of the proof of [10, Theorem 3.1]: we first perform a change of variables corresponding to a time contraction in order to relate $(T, \mu,[0, r])$ stabilizers to $(T / v, \mu / v,[0, r / v])$-stabilizers for $v>0$. We then study the stabilizability of a certain limit system, and this allows us to conclude the stabilizability of the original system for a certain $v>0$ large enough, thanks to the continuity result presented in the Appendix A.

\section{Step 1. Time contraction}

The system we consider is

$$
\begin{aligned}
& \dot{x}(t)=J_{d} x(t)-\alpha(t) B K x(t-\tau(t)), \\
& \alpha \in \mathcal{G}(T, \mu), \tau \in L^{\infty}\left(\mathbb{R}_{+},[0, r]\right) .
\end{aligned}
$$

For $v>0$, we define

$$
D_{d, v}=\operatorname{diag}\left(v^{d-1}, \ldots, v, 1\right)
$$

which satisfies the relations

$$
v D_{d, v}^{-1} J_{d} D_{d, v}=J_{d}, \quad D_{d, v} B=B
$$

Noting, for simplicity, $x(t)=x\left(t ; \tau, x_{0}, \alpha, K\right)$, and defining

$$
x_{v}(t)=D_{d, v}^{-1} x(v t)
$$

$x_{v}$ satisfies

$$
\frac{d}{d t} x_{v}(t)=J_{d} x_{v}(t)-\alpha(v t) v B K D_{d, v} x_{v}\left(t-\frac{\tau(v t)}{v}\right)
$$

and hence

$$
x_{v}(t)=x\left(t ; \frac{\tau(v \cdot)}{v}, D_{d, v}^{-1} x_{0}(v \cdot), \alpha_{v}, v K D_{d, v}\right)
$$

with $\alpha_{v}(t)=\alpha(v t)$, which is a $(T / v, \mu / v)$-signal. Thus $K$ is a $(T, \mu,[0, r])$-stabilizer for (3.2) if and only if $v K D_{d, v}$ is a $(T / v, \mu / v,[0, r / v])$-stabilizer. This equivalence is crucial in what follows: instead of looking for a $(T, \mu,[0, r])$-stabilizer for (3.2), we look for a $(T / v, \mu / v,[0, r / v])$-stabilizer for a certain $v>0$ large enough. The technique is thus to study a certain limit system obtained as $v \rightarrow+\infty$, obtain a stabilizer for this non-delayed system and then show that this stabilizer is actually a $(T / v, \mu / v,[0, r / v])$-stabilizer for a certain $v>0$ large enough.

Step 2. Limit system

We turn to the system

$$
\begin{aligned}
& \dot{x}(t)=J_{d} x(t)-\alpha_{\star}(t) B K x(t), \\
& \alpha_{\star} \in L^{\infty}\left(\mathbb{R}_{+},[\mu / T, 1]\right) .
\end{aligned}
$$

It has been proved in [10, Theorem 3.1], using a result from [11], that one can find $K \in$ $\mathcal{M}_{1, d}(\mathbb{R})$ and a positive definite matrix $S \in \mathcal{M}_{d}(\mathbb{R})$, both independent of the particular signal $\alpha_{\star} \in L^{\infty}\left(\mathbb{R}_{+},[\mu / T, 1]\right)$, such that (3.7) is globally uniformly exponentially stable and $V(x)=$ $x^{\mathrm{T}} S x$ decreases along all trajectories of (3.7), uniformly with respect to $\alpha_{\star}$. In particular, there exists a time $\sigma$ such that every trajectory of (3.7) starting in $B_{2}^{V}=\left\{x \in \mathbb{R}^{d} \mid V(x) \leq 2\right\}$ at time 0 lies in $B_{1}^{V}=\left\{x \in \mathbb{R}^{d} \mid V(x) \leq 1\right\}$ for every time larger than $\sigma$. 
Step 3. Study of (3.6) through the limit system.

We wish to deduce from the conclusion obtained in the previous step that (3.2) admits a $(T / v, \mu / v,[0, r / v])$-stabilizer for a certain $v>0$ large enough. We claim that, for some $v>0$ large enough, every trajectory of

$$
\begin{aligned}
& \dot{x}(t)=J_{d} x(t)-\alpha(t) B K x(t-\tau(t)), \\
& \alpha \in \mathcal{G}(T / v, \mu / v), \tau \in L^{\infty}\left(\mathbb{R}_{+},[0, r / v]\right),
\end{aligned}
$$

with initial condition $x_{0} \in \mathrm{C}^{0}\left([-r / v, 0], B_{2}^{V}\right)$ stays in $B_{1}^{V}$ for every time larger than $2 \sigma$. In particular, by homogeneity, this will imply that $K$ is a $(T / v, \mu / v,[0, r / v])$-stabilizer of (3.2) and thus $v^{-1} K D_{d, v}^{-1}$ is a $(T, \mu,[0, r])$-stabilizer, concluding the proof. To prove this, assume, by contradiction, that for every $n \in \mathbb{N}^{*}$ there exist $\tau_{n} \in L^{\infty}\left(\mathbb{R}_{+},[0, r / n]\right), x_{0}^{(n)} \in$ $\mathrm{C}^{0}\left([-r / n, 0], B_{2}^{V}\right), \alpha_{n} \in \mathcal{G}(T / n, \mu / n)$, and $t_{n} \in[2 \sigma, 4 \sigma]$ such that, for every $n \in \mathbb{N}^{*}$,

$$
x\left(t_{n} ; \tau_{n}, x_{0}^{(n)}, \alpha_{n}, K\right) \notin B_{1}^{V} .
$$

Up to the extraction of a subsequence, we can suppose that, as $n \rightarrow+\infty, t_{n} \rightarrow t_{\star} \in[2 \sigma, 4 \sigma]$,

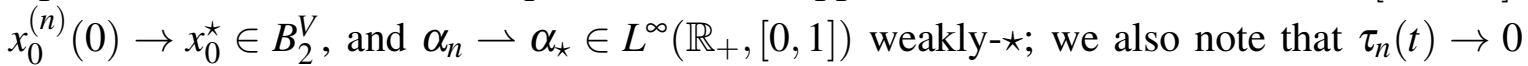
as $n \rightarrow+\infty$ uniformly on $t \in \mathbb{R}_{+}$. Then, applying Lemma A.1 proved in the Appendix A, we obtain that $x\left(t_{n} ; \tau_{n}, x_{0}^{(n)}, \alpha_{n}, K\right)$ converges to $x\left(t_{\star} ; 0, x_{0}^{\star}, \alpha_{\star}, K\right)$ as $n \rightarrow+\infty$. We also note that, by [10, Lemma 2.5], $\alpha_{\star}(t) \geq \mu / T$ almost everywhere in $\mathbb{R}_{+}$, and so, by our previous study of (3.7), since $t_{\star} \geq 2 \sigma$, by homogeneity, we have

$$
V\left(x\left(t_{\star} ; 0, x_{0}^{\star}, \alpha_{\star}, K\right)\right) \leq \frac{1}{2} .
$$

This contradicts (3.8), establishing the desired result.

\section{Main Result}

We now turn to the proof of our main result, Theorem 2.5. For a given stabilizable pair of matrices $(A, B) \in \mathcal{M}_{d}(\mathbb{R}) \times \mathcal{M}_{d, m}(\mathbb{R})$ and for given $T \geq \mu>0$ and $\tau_{0} \geq 0$, we wish to find an interval $\mathcal{T} \subset \mathbb{R}_{+}$of admissible perturbations around $\tau_{0}$ and a $(T, \mu, \mathcal{T})$-stabilizer for (2.2).

\section{Proof of Theorem 2.5.}

\section{Step 1. Reduction to a canonical form}

Notice that we may reduce the theorem to the case where $(A, B)$ is controllable, $m=1$, and all the eigenvalues of $A$ lie on the imaginary axis; this is detailed in Lemmas B.1, B.2, and B.3 in the Appendix B. We thus suppose from now on that $(A, B)$ is controllable, $m=1$, and $\Re(\lambda)=0$ for every eigenvalue $\lambda$ of $A$. We also reduce $(A, B)$ to a normal form with which it shall be easier to work.

Lemma 4.1. Suppose $(A, B) \in \mathcal{M}_{d}(\mathbb{R}) \times \mathbb{R}^{d}$ is a controllable pair and $\Re(\lambda)=0$ for every eigenvalue $\lambda$ of $A$. Then, up to a linear transformation of coordinates, (2.1) can be written as

$$
\begin{cases}\dot{x}_{0}(t)=J_{r_{0}} x_{0}(t)+\alpha(t) b^{0} u(t), & x_{0}(t) \in \mathbb{R}^{r_{0}}, \\ \dot{x}_{j}(t)=\left(\omega_{j} A^{(j)}+J_{r_{j}}^{C}\right) x_{j}(t)+\alpha(t) b^{j} u(t), & x_{j}(t) \in \mathbb{R}^{2 r_{j}}, \quad j=1, \ldots, h,\end{cases}
$$


where the spectrum of $A$ is $\sigma(A)=\left\{ \pm i \omega_{j}, j=j_{0}, j_{0}+1, \ldots, h\right\}$ with all the $\omega_{j} \geq 0$ distinct, $j_{0}=1$ if $0 \notin \sigma(A), j_{0}=0$ and $\omega_{0}=0$ otherwise; $r_{j}$ is the algebraic multiplicity of the eigenvalue $i \omega_{j}$ (with $r_{0}=0$ if $0 \notin \sigma(A)$ ); $J_{r_{0}}$ is the real Jordan block defined in (3.1); $J_{n}^{C} \in \mathcal{M}_{2 n}(\mathbb{R})$ is the Jordan block for complex eigenvalues,

$$
J_{n}^{C}=\left(\begin{array}{ccccccc}
0_{2 \times 2} & \mathrm{Id}_{2} & 0_{2 \times 2} & 0_{2 \times 2} & \cdots & 0_{2 \times 2} & 0_{2 \times 2} \\
0_{2 \times 2} & 0_{2 \times 2} & \operatorname{Id}_{2} & 0_{2 \times 2} & \cdots & 0_{2 \times 2} & 0_{2 \times 2} \\
0_{2 \times 2} & 0_{2 \times 2} & 0_{2 \times 2} & \operatorname{Id}_{2} & \cdots & 0_{2 \times 2} & 0_{2 \times 2} \\
0_{2 \times 2} & 0_{2 \times 2} & 0_{2 \times 2} & 0_{2 \times 2} & \cdots & 0_{2 \times 2} & 0_{2 \times 2} \\
\vdots & \vdots & \vdots & \vdots & \ddots & \vdots & \vdots \\
0_{2 \times 2} & 0_{2 \times 2} & 0_{2 \times 2} & 0_{2 \times 2} & \cdots & 0_{2 \times 2} & \operatorname{Id}_{2} \\
0_{2 \times 2} & 0_{2 \times 2} & 0_{2 \times 2} & 0_{2 \times 2} & \cdots & 0_{2 \times 2} & 0_{2 \times 2}
\end{array}\right)
$$

that is, $J_{n}^{C}=J_{n} \otimes \mathrm{Id}_{2}$ in terms of the Kronecker product; $A^{(j)}=\operatorname{diag}\left(A_{0}, \ldots, A_{0}\right) \in \mathcal{M}_{2 r_{j}}(\mathbb{R})$ with

$$
A_{0}=\left(\begin{array}{cc}
0 & 1 \\
-1 & 0
\end{array}\right)
$$

and $b^{0}$ and $b^{j}$ are respectively the vectors of $\mathbb{R}^{r_{0}}$ and $\mathbb{R}^{2 r_{j}}$ with all the coordinates equal to zero except the last one that is equal to one.

This lemma was proved in [10] during the proof of Theorem 3.2 therein; for the sake of completeness, we present briefly its proof in the Appendix B.

\section{Step 2. Time contraction}

We work from now on with system (4.1). Given $K \in \mathcal{M}_{1, d}\left(\mathbb{R}^{d}\right)$, we decompose $K$ in blocks as $K=\left(\begin{array}{llll}K_{0} & K_{1} & \cdots & K_{h}\end{array}\right)$ with $K_{0} \in \mathcal{M}_{1, r_{0}}(\mathbb{R}), K_{j} \in \mathcal{M}_{1,2 r_{j}}(\mathbb{R}), j=1, \ldots, h$, so that the feedback law $u(t)=-K x(t-\tau(t))$ is written as $u(t)=-K_{0} x_{0}(t-\tau(t))-\sum_{j=1}^{h} K_{j} x_{j}(t-$ $\tau(t))$. As in the proof of Theorem 3.1, we perform a change of time-space variables in the closed-loop system corresponding to a time contraction. Define

$$
\begin{aligned}
& y_{0}(t)=D_{r_{0}, v}^{-1} x_{0}(v t), \\
& y_{j}(t)=\left(D_{r_{j}, v}^{C}\right)^{-1} e^{-v t \omega_{j} A^{(j)}} x_{j}(v t), \quad j=1, \ldots, h,
\end{aligned}
$$

with $D_{n, v}$ as in (3.3), satisfying (3.4), and

$$
D_{n, v}^{C}=D_{n, v} \otimes \operatorname{Id}_{2}=\operatorname{diag}\left(v^{n-1}, v^{n-1}, \ldots, v, v, 1,1\right) \in \mathcal{M}_{2 n}(\mathbb{R}),
$$

which satisfies

$$
v\left(D_{r_{j}, v}^{C}\right)^{-1} J_{r_{j}}^{C} D_{r_{j}, v}^{C}=J_{r_{j}}^{C}, \quad D_{r_{j}, v}^{C} b^{j}=b^{j}, \quad j=1, \ldots, h .
$$

Then $y_{0}, y_{1}, \ldots, y_{h}$ satisfy

$$
\left\{\begin{aligned}
\dot{y}_{0}(t)= & J_{r_{0}} y_{0}(t)-\alpha_{v}(t) b^{0}\left[K_{0, v} y_{0}\left(t-\frac{\tau(v t)}{v}\right)+\sum_{\ell=1}^{h} K_{\ell, v} e^{(v t-\tau(v t)) \omega_{\ell} A^{(\ell)}} y_{\ell}\left(t-\frac{\tau(v t)}{v}\right)\right], \\
\dot{y}_{j}(t)= & J_{r_{j}}^{C} y_{j}(t)-\alpha_{v}(t) e^{-v t \omega_{j} A^{(j)}} b^{j}\left[K_{0, v} y_{0}\left(t-\frac{\tau(v t)}{v}\right)+\right. \\
& \left.+\sum_{\ell=1}^{h} K_{\ell, v} e^{(v t-\tau(v t)) \omega_{\ell} A^{(\ell)}} y_{\ell}\left(t-\frac{\tau(v t)}{v}\right)\right], \quad j=1, \ldots, h,
\end{aligned}\right.
$$


with $\alpha_{v}(t)=\alpha(v t), K_{0, v}=v K_{0} D_{r_{0}, v}, K_{\ell, v}=v K_{\ell} D_{r_{\ell}, v}^{C}$ for $\ell=1, \ldots, h$, and where we use that $A^{(j)} D_{r_{j}, v}^{C}=D_{r_{j}, v}^{C} A^{(j)}$ and $A^{(j)} J_{r_{j}}^{C}=J_{r_{j}}^{C} A^{(j)}$ for $j=1, \ldots, h$. This shows that the gain $K=\left(\begin{array}{llll}K_{0} & K_{1} & \cdots & K_{h}\end{array}\right)$ is a $(T, \mu, \mathcal{T})$-stabilizer for (4.1) if and only if the gain $K_{v}=$ $\left(\begin{array}{llll}K_{0, v} & K_{1, v} & \cdots & K_{h, v}\end{array}\right)$ is a $(T / v, \mu / v, \mathcal{T} / v)$-stabilizer for (4.2), where $\mathcal{T} / v=\{t / v \mid t \in$ $\mathcal{T}\}$.

\section{Step 3. Choice of the feedback family}

We turn to the problem of finding a neighborhood $\mathcal{T}$ of $\tau_{0}$ in $\mathbb{R}_{+}$and a $(T / v, \mu / v, \mathcal{T} / v)$ stabilizer for (4.2) for a certain $v>0$, which will imply the theorem. We shall look for such a stabilizer $K_{v}$ under a particular form. We write $b_{0}=\left(\begin{array}{ll}0 & 1\end{array}\right)^{\mathrm{T}}$ and we take $K_{v}=$ $\left(\begin{array}{llll}K_{0, v} & K_{1, v} & \cdots & K_{h, v}\end{array}\right)$ with

$$
\begin{array}{ll}
K_{0, v}=\mathcal{K}_{0}, & \mathcal{K}_{0}=\left(\begin{array}{lll}
k_{1}^{0} & \cdots & k_{r_{0}}^{0}
\end{array}\right) \in \mathcal{M}_{1, r_{0}}(\mathbb{R}) \\
K_{j, v}=\mathcal{K}_{j} \otimes b_{0}^{\mathrm{T}} e^{\tau_{0} \omega_{j} A_{0}}, & \mathcal{K}_{j}=\left(\begin{array}{lll}
k_{1}^{j} & \cdots & k_{r_{j}}^{j}
\end{array}\right) \in \mathcal{M}_{1, r_{j}}(\mathbb{R}), \quad j=1, \ldots, h .
\end{array}
$$

Now, since $A^{(\ell)}=\operatorname{Id}_{r_{\ell}} \otimes A_{0}$, we have, for $\ell=1, \ldots, h$, that

$$
\begin{aligned}
K_{\ell, v} e^{(v t-\tau(v t)) \omega_{\ell} A^{(\ell)}} & =\mathcal{K}_{\ell} \otimes b_{0}^{\mathrm{T}} e^{\left(v t-\tau(v t)+\tau_{0}\right) \omega_{\ell} A_{0}}= \\
& =\mathcal{K}_{\ell} \otimes b_{0}^{\mathrm{T}} e^{v t \omega_{\ell} A_{0}}+\mathcal{K}_{\ell} \otimes\left[b_{0}^{\mathrm{T}} e^{v t \omega_{\ell} A_{0}}\left(e^{-\left(\tau(v t)-\tau_{0}\right) \omega_{\ell} A_{0}}-\mathrm{Id}_{2}\right)\right] .
\end{aligned}
$$

Noting $\tilde{b}^{j} \in \mathbb{R}^{r_{j}}$ the vector with all coordinates equal to zero except the last one that is equal to one, we have $b^{j}=\tilde{b}^{j} \otimes b_{0}$, and thus $e^{-v t \omega_{j} A^{(j)}} b^{j}=\tilde{b}_{j} \otimes e^{-v t \omega_{j} A_{0}} b_{0}$. We finally write, for $j, \ell \in\{1, \ldots, h\}$,

$$
\begin{aligned}
& C_{00}^{(v)}(t)=\alpha_{v}(t) \\
& C_{0 j}^{(v)}(t)=\alpha_{v}(t) b_{0}^{\mathrm{T}} e^{v t \omega_{j} A_{0}} \\
& C_{j 0}^{(v)}(t)=\alpha_{v}(t) e^{-v t \omega_{j} A_{0}} b_{0} \\
& C_{j \ell}^{(v)}(t)=\alpha_{v}(t) e^{-v t \omega_{j} A_{0}} b_{0} b_{0}^{\mathrm{T}} e^{v t \omega_{j} A_{0}} \\
& P_{00}^{(v)}(t)=P_{j 0}^{(v)}(t)=0 \\
& P_{0 j}^{(v)}(t)=\alpha_{v}(t) b_{0}^{\mathrm{T}} e^{v t \omega_{j} A_{0}}\left[e^{-\left(\tau(v t)-\tau_{0}\right) \omega_{j} A_{0}}-\mathrm{Id}_{2}\right], \\
& P_{j \ell}^{(v)}(t)=\alpha_{v}(t) e^{-v t \omega_{j} A_{0}} b_{0} b_{0}^{\mathrm{T}} e^{v t \omega_{\ell} A_{0}}\left[e^{\left.-\left(\tau(v t)-\tau_{0}\right) \omega_{\ell} A_{0}-\mathrm{Id}_{2}\right]}\right.
\end{aligned}
$$

and thus system (4.2) can be written under the form

$$
\left\{\begin{array}{l}
\dot{y}_{0}(t)=J_{r_{0}} y_{0}(t)-\sum_{\ell=0}^{h}\left[b^{0} \mathcal{K}_{\ell} \otimes\left(C_{0 \ell}^{(v)}(t)+P_{0 \ell}^{(v)}(t)\right)\right] y_{\ell}\left(t-\frac{\tau(v t)}{v}\right), \\
\dot{y}_{j}(t)=J_{r_{j}}^{C} y_{j}(t)-\sum_{\ell=0}^{h}\left[\tilde{b}^{j} \mathcal{K}_{\ell} \otimes\left(C_{j \ell}^{(v)}(t)+P_{j \ell}^{(v)}(t)\right)\right] y_{\ell}\left(t-\frac{\tau(v t)}{v}\right), \quad j=1, \ldots, h .
\end{array}\right.
$$

We can arrange all the matrices $C_{j \ell}^{(v)}$ in a $\left(2 h+1-j_{0}\right) \times\left(2 h+1-j_{0}\right)$ symmetric matrix and all the matrices $P_{j \ell}^{(v)}$ in a $\left(2 h+1-j_{0}\right) \times\left(2 h+1-j_{0}\right)$ matrix respectively as

$$
C^{(v)}(t)=\left(C_{j \ell}^{(v)}(t)\right)_{j_{0} \leq j, \ell \leq h}, \quad P^{(v)}(t)=\left(P_{j \ell}^{(v)}(t)\right)_{j_{0} \leq j, \ell \leq h} .
$$


We take from now on $\mathcal{T}$ under the form $\mathcal{T}=\left[\tau_{0}-r, \tau_{0}+r\right] \cap \mathbb{R}_{+}$for a certain $r>0$ to be chosen, and so

$$
\begin{aligned}
\left\|P_{j \ell}^{(v)}(t)\right\| \leq\left\|e^{-\left(\tau(v t)-\tau_{0}\right) \omega_{j} A_{0}}-\operatorname{Id}_{2}\right\| & = \\
& =\sqrt{2\left[1-\cos \left(\left(\tau(v t)-\tau_{0}\right) \omega_{j}\right)\right]} \leq\left|\left(\tau(v t)-\tau_{0}\right) \omega_{j}\right| \leq r \Omega
\end{aligned}
$$

with $\Omega=\max \left\{\omega_{j} \mid j=j_{0}, \ldots, h\right\}$.

Step 4. Limit system

We wish to study (4.5) through a limit system, as we did with (3.6) in Theorem 3.1. The stability result for the limit system is given in the following lemma, proved later on in Appendix B.

Lemma 4.2. Consider the system

$$
\left\{\begin{array}{l}
\dot{y}_{0}(t)=J_{r_{0}} y_{0}(t)-\sum_{\ell=0}^{h}\left[b^{0} \mathcal{K}_{\ell} \otimes\left(C_{0 \ell}(t)+P_{0 \ell}(t)\right)\right] y_{\ell}(t), \\
\dot{y}_{j}(t)=J_{r_{j}}^{C} y_{j}(t)-\sum_{\ell=0}^{h}\left[\tilde{b}^{j} \mathcal{K}_{\ell} \otimes\left(C_{j \ell}(t)+P_{j \ell}(t)\right)\right] y_{\ell}(t), \quad j=1, \ldots, h,
\end{array}\right.
$$

where $y_{0} \in \mathbb{R}^{r_{0}}, y_{j} \in \mathbb{R}^{2 r_{j}}, J_{n}$ and $J_{n}^{C}$ are the Jordan blocks defined above, $b^{0}$ and $\tilde{b}^{j}$ are the vectors defined above, $\mathcal{K}_{j} \in \mathcal{M}_{1, r_{j}}(\mathbb{R})$ are constant matrices, $j=j_{0}, \ldots, h, C_{\star}, P_{\star} \in$ $L^{\infty}\left(\mathbb{R}_{+}, \mathcal{M}_{2 h+1-j_{0}}(\mathbb{R})\right)$ and the $2 \times 2$ time-dependent matrices $C_{j \ell}, P_{j \ell}, 1 \leq j, \ell \leq h$, the $(1-$ $\left.j_{0}\right) \times 2$ time-dependent matrices $C_{0 \ell}, P_{0 \ell}$, the $2 \times\left(1-j_{0}\right)$ time-dependent matrices $C_{j 0}, P_{j 0}$ and the signals $C_{00}, P_{00}$ are defined by the relations

$$
C_{\star}(t)=\left(C_{j \ell}(t)\right)_{j_{0} \leq j, \ell \leq h}, \quad P_{\star}(t)=\left(P_{j \ell}(t)\right)_{j_{0} \leq j, \ell \leq h},
$$

and we also assume that

$$
\left\|P_{j \ell}(t)\right\| \leq r \Omega, \quad \text { for almost every } t \in \mathbb{R}_{+}, \forall j, \ell \in\left\{j_{0}, \ldots, h\right\} .
$$

We write $y=\left(\begin{array}{llll}y_{0}^{\mathrm{T}} & y_{1}^{\mathrm{T}} & \cdots & y_{h}^{\mathrm{T}}\end{array}\right)^{\mathrm{T}}$.

Let $\xi>0$. Then there exist $C \geq 1, \gamma>0, r>0$, and $\mathcal{K}_{j} \in \mathcal{M}_{1, r_{j}}(\mathbb{R}), j=j_{0}, \ldots, h$, such that, for every symmetric matrix $C_{\star} \in L^{\infty}\left(\mathbb{R}_{+}, \mathcal{M}_{2 h+1-j_{0}}(\mathbb{R})\right)$ satisfying $C_{\star}(t) \geq \xi \operatorname{Id}_{2 h+1-j_{0}}$ almost everywhere, every $P_{\star} \in L^{\infty}\left(\mathbb{R}_{+}, \mathcal{M}_{2 h+1-j_{0}}(\mathbb{R})\right)$ satisfying (4.9) and every solution $y$ of (4.7), we have

$$
\|y(t)\| \leq C e^{-\gamma t}\|y(0)\|, \quad \forall t \geq 0
$$

Step 5. Study of (4.5) through the limit system

To conclude the proof, we deduce the stability of (4.5) from that of (4.7) in the same way as we did in the proof of Theorem 3.1. Take $T \geq \mu>0$ and $\tau_{0} \geq 0$. By [10, Lemma 2.5], there exists $\xi>0$ depending only on $T, \mu$ and $\omega_{j}, j=j_{0}, \ldots, h$, such that, for any $\alpha \in \mathcal{G}(T, \mu)$ and any $v>0$, the time-dependent matrix $C^{(v)}$ constructed from $\alpha$ as in (4.4) and (4.6) is in $L^{\infty}\left(\mathbb{R}_{+}, \mathcal{M}_{2 h+1-j_{0}}(\mathbb{R})\right)$ and satisfies

$$
\int_{t}^{t+\frac{T}{v}} C^{(v)}(s) d s \geq \xi \frac{T}{v} \operatorname{Id}_{2 h+1-j_{0}}
$$


For this $\xi>0$, take $C \geq 1, \gamma>0, r>0$, and $\mathcal{K}_{j} \in \mathcal{M}_{1, r_{j}}(\mathbb{R})$ as in Lemma 4.2. Set $\mathcal{T}=$ $\left[\tau_{0}-r, \tau_{0}+r\right] \cap \mathbb{R}_{+}$and construct $K=\left(\begin{array}{lll}K_{0} & \cdots & K_{h}\end{array}\right)$ from the $\mathcal{K}_{j}, j=j_{0}, \ldots, h$ as in (4.3). We want to show that, for $v>0$ large enough, $K$ is a $(T / v, \mu / v, \mathcal{T} / v)$-stabilizer for (4.2), and this will conclude the proof by the conclusion of Step 2 .

Note that, by Lemma 4.2, there exists a time $\sigma>0$ depending only on $C$ and $\gamma$ such that, for every trajectory $y$ of (4.7) starting in $B_{2}=\left\{x \in \mathbb{R}^{d} \mid\|x\| \leq 2\right\}$ at time 0 lies in $B_{1}=\left\{x \in \mathbb{R}^{d} \mid\|x\| \leq 1\right\}$ for every time larger than $\sigma$. We claim that, for some $v>0$ large enough, for every $\alpha \in \mathcal{G}(T / v, \mu / v)$, every $\tau \in L^{\infty}\left(\mathbb{R}_{+}, \mathcal{T} / v\right)$ and every initial condition $y^{0} \in \mathrm{C}^{0}\left([-R / v, 0], B_{2}\right)$, with $R=\sup \mathcal{T}$, the solution $y$ of (4.5), with $C^{(v)}$ and $P^{(v)}$ given by (4.4) and (4.6), stays in $B_{1}$ for every time larger than $2 \sigma$. This will show, by homogeneity, that $K$ is a $(T / v, \mu / v, \mathcal{T} / v)$-stabilizer for (4.2).

Assume, by contradiction, that for every $n \in \mathbb{N}^{*}$ there exist $\tau_{n} \in L^{\infty}\left(\mathbb{R}_{+}, \mathcal{T} / n\right), y_{n}^{0} \in$ $\mathcal{C}^{0}\left([-R / n, 0], B_{2}\right), \alpha_{n} \in \mathcal{G}(T / n, \mu / n)$, and $t_{n} \in[2 \sigma, 4 \sigma]$ such that, for every $n \in \mathbb{N}^{*}$, the solution $y_{n}$ of (4.5), with $C^{(n)}$ and $P^{(n)}$ given by (4.4) and (4.6), satisfies

$$
y_{n}\left(t_{n}\right) \notin B_{1} \text {. }
$$

Up to the extraction of a subsequence, we can suppose that

$$
\begin{aligned}
& \lim _{n \rightarrow \infty} t_{n}=t_{\star} \in[2 \sigma, 4 \sigma], \\
& \lim _{n \rightarrow \infty} y_{n}^{0}(0)=y_{\star}^{0} \in B_{2} \text {, } \\
& \lim _{n \rightarrow \infty} C^{(n)}=C_{\star} \in L^{\infty}\left(\mathbb{R}_{+}, \mathcal{M}_{2 h+1-j_{0}}(\mathbb{R})\right) \quad \text { weakly- } \star, \\
& \lim _{n \rightarrow \infty} P^{(n)}=P_{\star} \in L^{\infty}\left(\mathbb{R}_{+}, \mathcal{M}_{2 h+1-j_{0}}(\mathbb{R})\right) \quad \text { weakly- } \star,
\end{aligned}
$$

and we also note that $\tau_{n}(t) \rightarrow 0$ uniformly on $t \in \mathbb{R}_{+}$as $n \rightarrow+\infty$. Then, by Lemma A.1, $y_{n}$ converges to the solution $y_{\star}$ of (4.7) associated to $C_{\star}, P_{\star}$ and with initial condition $y_{\star}^{0}$, uniformly on compact time intervals, and in particular $y_{n}\left(t_{n}\right) \rightarrow y_{\star}\left(t_{\star}\right)$. By [10, Lemma 2.5], we have $C_{\star}(t) \geq \xi \operatorname{Id}_{2 h+1-j_{0}}$ for almost every $t$ and, since $\left\|P_{j \ell}^{(n)}(t)\right\| \leq r \Omega$ for every $j, \ell \in\left\{j_{0}, \ldots, h\right\}$ and almost every $t \in \mathbb{R}_{+}$, we have, by the lower semicontinuity of the norm of $L^{\infty}\left(\mathbb{R}_{+}, \mathcal{M}_{2 h+1-j_{0}}(\mathbb{R})\right)$, that $\left\|P_{j \ell}(t)\right\| \leq r \Omega$ for every $j, \ell \in\left\{j_{0}, \ldots, h\right\}$ and almost every $t \in \mathbb{R}_{+}$, where $P_{j \ell}$ is obtained from $P_{\star}$ by (4.8). Thus we are under the hypotheses of Lemma 4.2 , and so our previous discussion shows us that $y_{\star}$ remains in $B_{1}$ for every time larger than $\sigma$; by homogeneity, $\left\|y_{\star}\left(t_{\star}\right)\right\| \leq 1 / 2$ since $t_{\star} \geq 2 \sigma$. This contradicts (4.10), establishing the desired result.

\section{Further Discussion}

We proved that persistently excited linear systems can be stabilized by a delayed feedback law when the uncontrolled dynamics of the system is given by a matrix $A$ whose eigenvalues have all non-positive real part and when the delay varies in an interval around a constant value $\tau_{0}$, with the feedback matrix $K$ depending on the matrices $A, B$, on the constants $T$ and $\mu$ of the condition of persistence of excitation and on the reference delay $\tau_{0}$. This is a generalization of [10, Theorem 3.2], originally proved for the non-delayed case.

The technique of the proof consists on adapting the time-contraction argument of [10, Theorem 3.2] to the delayed case. Indeed, the time contraction also contracts the delay, reducing its 
effect, and the limit system obtained in the time-contraction procedure is the same as in [10], except for the new terms $P_{j \ell}$, which are treated as perturbations of the limit system of [10].

It is actually by treating these terms $P_{j \ell}$ as perturbations that we arrive to the construction of the delay neighborhood $\mathcal{T}$ around $\tau_{0}$ where we can guarantee stabilizability. Note that the terms $P_{j \ell}$ do not appear in the limit system obtained when $A=J_{d}$ in Theorem 3.1, since they depend on the eigenvalues $i \omega_{j}$, and this is the reason why we can obtain a $(T, \mu, \mathcal{T})$-stabilizer for any bounded $\mathcal{T} \subset \mathbb{R}_{+}$when $A=J_{d}$ in Theorem 3.1.

This is a fundamental difference between Theorems 3.1 and 2.5 which we would like to highlight: in Theorem 3.1, stabilization can be achieved for any bounded set $\mathcal{T} \subset \mathbb{R}_{+}$where the delay takes its values, whereas in Theorem $2.5 \mathcal{T}$ is chosen as $\mathcal{T}=\left[\tau_{0}-r, \tau_{0}+r\right] \cap \mathbb{R}_{+}$, a perturbation around the constant value $\tau_{0}$.

A natural question is then to study if Theorem 2.5 might not be generalized for any bounded set $\mathcal{T}$ instead of considering only perturbations around $\tau_{0}$. This is actually not possible, as shown in the following example, where we take $\alpha$ identically equal to one, i.e., the control is completely active the whole time.

Example 5.1. Consider the control system

$$
\dot{x}=A x+B u
$$

with

$$
A=\left(\begin{array}{cc}
0 & 1 \\
-1 & 0
\end{array}\right), \quad B=\left(\begin{array}{l}
0 \\
1
\end{array}\right)
$$

and submitted to the feedback law

$$
u(t)=-K x(t-\tau(t))
$$

This control system does not depend on a persistently exciting signal $\alpha$, but, in order to keep the notations we used previously, we shall consider it as a persistently excited system with constants $T=\mu$, so that $\mathcal{G}(T, \mu)=\mathcal{G}(T, T)$ reduces to the class containing only the constant signal identically equal to one. We want to prove that the conclusion of Theorem 3.1 does not hold for (5.1), that is, we want to show that there exists a bounded interval $\mathcal{T}$ for which (5.1) with the feedback (5.2) does not admit a $(T, T, \mathcal{T})$-stabilizer. Obviously, this also implies the non-existence of a $(T, \mu, \mathcal{T})$-stabilizer for every $\mu \in(0, T]$ since such a stabilizer would be in particular a $(T, T, \mathcal{T})$-stabilizer.

We claim that (5.1) with the feedback (5.2) does not admit a $(T, T,[0,2 \pi])$-stabilizer. In order to simplify our analysis, we shall consider only constant-in-time delays in the interval $[0,2 \pi]$, which allow us to apply the techniques of stability analysis for delayed systems presented in [21].

The closed-loop system obtained from (5.1) with the feedback (5.2) and a constant delay $\tau \in[0,2 \pi]$ is

$$
\dot{x}(t)=A x(t)-B K x(t-\tau) .
$$

According to [21, Proposition 1.6], the stability of (5.3) can be studied through the complex roots $\lambda$ of the characteristic equation

$$
\operatorname{det}\left(\lambda \operatorname{Id}_{2}-A+B K e^{-\lambda \tau}\right)=0
$$

the origin of (5.3) is exponentially stable if and only if all the roots $\lambda$ of (5.4) satisfy $\Re(\lambda)<$ 0 , and exponential stability and asymptotic stability are also equivalent in this case. 
Writing $K=\left(\begin{array}{ll}k_{1} & k_{2}\end{array}\right)$, the characteristic equation (5.4) is

$$
\lambda^{2}+k_{2} \lambda e^{-\lambda \tau}+1+k_{1} e^{-\lambda \tau}=0
$$

We now want to show that, for every $K \in \mathcal{M}_{1,2}(\mathbb{R})$, there exists $\tau \in[0,2 \pi]$ such that (5.5) admits a root $\lambda$ with $\Re(\lambda) \geq 0$. As remarked in [21, Theorem 1.15], by the continuity of the real part of the largest eigenvalue with respect to the delay, this study is reduced to the problem of finding a delay $\tau \in[0,2 \pi]$ such that (5.5) admits a root $\lambda$ with $\Re(\lambda)=0$.

The feedback $K=0$ obviously does not stabilize the system to the origin, and so we suppose from now on that $k_{1}$ and $k_{2}$ are not simultaneously zero. We look for a certain $\tau \in[0,2 \pi]$ and a root $\lambda=i \omega$ of (5.5) with $\omega \in \mathbb{R}$. We thus want $\omega$ to satisfy

$$
\left\{\begin{array}{r}
1-\omega^{2}+k_{1} \cos (\tau \omega)+k_{2} \omega \sin (\tau \omega)=0 \\
-k_{1} \sin (\tau \omega)+k_{2} \omega \cos (\tau \omega)=0 .
\end{array}\right.
$$

This is equivalent to

$$
\left\{\begin{aligned}
\sin \theta & =\frac{k_{2} \omega\left(\omega^{2}-1\right)}{k_{2}^{2} \omega^{2}+k_{1}^{2}}, \\
\cos \theta & =\frac{k_{1}\left(\omega^{2}-1\right)}{k_{2}^{2} \omega^{2}+k_{1}^{2}}, \\
\theta & =\tau \omega
\end{aligned}\right.
$$

and such a system can only have a solution if $\sin ^{2} \theta+\cos ^{2} \theta=1$, which is the case if and only if $\left(\omega^{2}-1\right)^{2}=k_{2}^{2} \omega^{2}+k_{1}^{2}$. This last equation is a polynomial in $\omega^{2}$ of degree 2 , whose solutions can be computed explicitly as

$$
\omega^{2}=\frac{1}{2}\left[2+k_{2}^{2} \pm \sqrt{\left(2+k_{2}^{2}\right)^{2}-4\left(1-k_{1}^{2}\right)}\right] .
$$

We consider from now on the solution

$$
\omega=\sqrt{\frac{2+k_{2}^{2}+\sqrt{\left(2+k_{2}^{2}\right)^{2}-4\left(1-k_{1}^{2}\right)}}{2}} .
$$

Note that $\omega$ is well-defined in $\mathbb{R}$ since $\left(2+k_{2}^{2}\right)^{2}>4\left(1-k_{1}^{2}\right)$ for any $K \in \mathcal{M}_{1,2}(\mathbb{R}) \backslash\{0\}$, and that $\omega \geq 1$. With this $\omega$, we can thus find $\theta \in[0,2 \pi]$ such that (5.6) is satisfied, and so $\tau=\theta / \omega \in[0,2 \pi]$ since $\omega \geq 1$. Since the constructed $(\theta, \tau, \omega)$ satisfies (5.6), (5.5) is hence satisfied for $\tau$ and $\lambda=i \omega$, and thus (5.3) is not asymptotically stable. Hence (5.1) admits no $(T, T,[0,2 \pi])$-stabilizer.

Note that we could replace $[0,2 \pi]$ in Example 5.1 for any other interval $\mathcal{T} \subset \mathbb{R}_{+}$with length greater than or equal $2 \pi$, and so we conclude that (5.1) does not admit a $(T, \mu, \mathcal{T})$-stabilizer if $\mathcal{T}$ contains an interval with length greater than or equal $2 \pi$.

The value $2 \pi$ obtained in these computations comes from the fact that the dynamics given by the matrix $A$ we chose correspond to rotations around the origin with unitary angular velocity, and $2 \pi$ is the total time that a solution of $\dot{x}=A x$ takes to make a complete turn around the origin. If we choose $A$ as

$$
A=\left(\begin{array}{cc}
0 & \omega_{0} \\
-\omega_{0} & 0
\end{array}\right)
$$


for $\omega_{0} \neq 0$, then the same computations as in Example 5.1 show that no $(T, \mu, \mathcal{T})$-stabilizer can exist for (5.1) if $\mathcal{T}$ contains an interval of length at least $\frac{2 \pi}{\omega_{0}}$. In particular, this gives a link between an upper bound on the maximal length of an interval contained in $\mathcal{T}$ for which a $(T, \mu, \mathcal{T})$-stabilizer exists and the eigenvalues of $A$ on the imaginary axis.

This example shows that the fundamental difference in the statement of Theorems 3.1 and 2.5 concerning the choice of the set $\mathcal{T}$ actually comes from the dynamics of the system itself, and that no improvement of Theorem 2.5 as good as Theorem 3.1 can be obtained.

\section{A Appendix: A Continuity Result for Delayed Systems}

We show here a continuity result of the solution of a delayed system with respect to its parameters, in the spirit of [7, Proposition 21], which is used in the proof of Theorems 3.1 and 2.5. We place ourselves in a more general setting than (2.2), considering the system

$$
\dot{x}(t)=A x(t)+B(t) x(t-\tau(t)),
$$

where $\tau \in L^{\infty}\left(\mathbb{R}_{+},[0, r]\right)$, and $B \in L^{\infty}\left(\mathbb{R}_{+}, \mathcal{M}_{d}(\mathbb{R})\right)$ is a time-dependent matrix. We remark that (A.1) satisfies the Carathéodory conditions for delayed equations, and so, for fixed $\tau$ and $B$ and for any given initial condition $x_{0} \in \mathfrak{C}^{0}\left([-r, 0], \mathbb{R}^{d}\right),($ A.1 $)$ admits a unique continuous solution $x$ defined on $[-r,+\infty)$, which we denote by $x(t)=x\left(t ; \tau, x_{0}, B\right)$; this solution is absolutely continuous on $\mathbb{R}_{+}$, coincides with $x_{0}$ on $[-r, 0]$, and satisfies (A.1) for almost every $t \in \mathbb{R}_{+}$. Our continuity result can then be stated as follows.

Lemma A.1. Let $\left(\tau_{n}\right)_{n \in \mathbb{N}^{*}}$ be a sequence on $L^{\infty}\left(\mathbb{R}_{+},[0, r]\right)$ such that $\tau_{n}(t) \rightarrow 0$ as $n \rightarrow+\infty$ uniformly on $\mathbb{R}_{+}$. Suppose $\left(x_{0}^{(n)}\right)_{n \in \mathbb{N}^{*}}$ is a sequence of functions in $\mathrm{C}^{0}\left([-r, 0], \mathbb{R}^{d}\right)$ and $\left(B_{n}\right)_{n \in \mathbb{N}^{*}}$ a bounded sequence on $L^{\infty}\left(\mathbb{R}_{+}, \mathcal{M}_{d}(\mathbb{R})\right)$ satisfying

1. $\lim _{n \rightarrow+\infty} x_{0}^{(n)}(0)=x_{0}^{\star}$ for a certain $x_{0}^{\star} \in \mathbb{R}^{d}$;

2. there exists $\Lambda>0$ such that $\left\|x_{0}^{(n)}(t)\right\| \leq \Lambda$ for all $n \in \mathbb{N}^{*}$ and all $t \in[-r, 0]$;

3. $B_{n} \underset{n \rightarrow+\infty}{\longrightarrow} B_{\star}$ weakly-^for a certain $B_{\star} \in L^{\infty}\left(\mathbb{R}_{+}, \mathcal{M}_{d}(\mathbb{R})\right)$.

Then $x\left(t ; \tau_{n}, x_{0}^{(n)}, B_{n}\right) \underset{n \rightarrow+\infty}{\longrightarrow} x\left(t ; 0, x_{0}^{\star}, B_{\star}\right)$, uniformly on compact time intervals in $\mathbb{R}_{+}$.

Proof. We can extend $B_{\star}$ outside $\mathbb{R}_{+}$to the whole real line in such a way that this extension is an element of $L^{\infty}\left(\mathbb{R}, \mathcal{M}_{d}(\mathbb{R})\right)$. We fix such an extension, so that $x\left(\cdot ; 0, x_{0}^{\star}, B_{\star}\right)$ is absolutely continuous in $\mathbb{R}$ and satisfies (A.1) for almost every $t \in \mathbb{R}$; note that this is possible since $x\left(\cdot ; 0, x_{0}^{\star}, B_{\star}\right)$ is the solution of a non-delayed system. For simplicity, we shall note $x_{n}(t)=x\left(t ; \tau_{n}, x_{0}^{(n)}, B_{n}\right)$ and $x_{\star}(t)=x\left(t ; 0, x_{0}^{\star}, B_{\star}\right)$. We also note by $M$ an upper bound on $\left\|B_{n}\right\|_{L^{\infty}\left(\mathbb{R}_{+}, \mathcal{M}_{d}(\mathbb{R})\right)}$ and $r_{n}=\sup _{t \in \mathbb{R}_{+}} \tau_{n}(t)$, and, by the uniform convergence of $\tau_{n}$ to 0 , we have that $r_{n} \rightarrow 0$ as $n \rightarrow+\infty$.

Define $e_{n}(t)=x_{n}(t)-x_{\star}(t)$ for $t \geq-r$. Then, for $t \geq 0, e_{n}$ satisfies

$$
\dot{e}_{n}(t)=A e_{n}(t)+B_{n}(t) e_{n}\left(t-\tau_{n}(t)\right)+f_{n}(t)
$$

with $f_{n}$ given by $f_{n}(t)=B_{n}(t)\left(x_{\star}\left(t-\tau_{n}(t)\right)-x_{\star}(t)\right)+\left(B_{n}(t)-B_{\star}(t)\right) x_{\star}(t)$. 
Since $x_{\star}$ is continuous, it follows from Lebesgue's Dominated Convergence Theorem that

$$
\lim _{n \rightarrow+\infty} \int_{0}^{t} B_{n}(s)\left(x_{\star}\left(s-\tau_{n}(s)\right)-x_{\star}(s)\right) d s=0
$$

for every $t \geq 0$. By the weak- $\star$ convergence of $\left(B_{n}\right)$, we have that

$$
\lim _{n \rightarrow+\infty} \int_{0}^{t}\left(B_{n}(s)-B_{\star}(s)\right) x_{\star}(s) d s=0,
$$

and so $f_{n}$ satisfies

$$
\lim _{n \rightarrow+\infty} \int_{0}^{t} f_{n}(s) d s=0
$$

for every $t \geq 0$. Letting $F_{n}(t)=\int_{0}^{t} f_{n}(s) d s$, this shows that $F_{n}(t) \underset{n \rightarrow+\infty}{\longrightarrow} 0$ for every $t \geq$ 0 . This limit is uniform on compact time intervals in $\mathbb{R}_{+}$. Indeed, let $T>0$ and $X_{\star}=$ $\sup _{t \in[-r, T]}\left\|x_{\star}(t)\right\|$; we thus see that $\left\|f_{n}(t)\right\| \leq 2 M X_{\star}$ and so $\left\|F_{n}(t)\right\| \leq 2 M X_{\star} T$ for every $t \in[0, T]$. Furthermore, for $0 \leq t_{1}<t_{2} \leq T$, we have

$$
\left\|F_{n}\left(t_{2}\right)-F_{n}\left(t_{1}\right)\right\| \leq \int_{t_{1}}^{t_{2}}\left\|f_{n}(s)\right\| d s \leq 2 M X_{\star}\left(t_{2}-t_{1}\right)
$$

and hence $\left(F_{n}\right)$ is equicontinuous. Thus, by Arzelà-Ascoli Theorem, the closure of $\left\{F_{n} \mid n \in\right.$ $\left.\mathbb{N}^{*}\right\}$ is a compact subset of $\mathcal{C}^{0}\left([0, T], \mathbb{R}^{d}\right)$ with the topology of the uniform convergence, and so this set has at least one limit point; it has exactly one, for, if it had two distinct limit points, this would contradict the fact that $\left(F_{n}(t)\right)_{n \in \mathbb{N}^{*}}$ tends pointwise to 0 , and so the sequence $\left(F_{n}\right)_{n \in \mathbb{N}^{*}}$ converges uniformly to 0 in $[0, T]$.

Integrating (A.2) from 0 to $t \geq 0$, we obtain

$$
e_{n}(t)=e_{n}(0)+F_{n}(t)+\int_{0}^{t} A e_{n}(s) d s+\int_{0}^{t} B_{n}(s) e_{n}\left(s-\tau_{n}(s)\right) d s,
$$

which gives us the estimate

$$
\left\|e_{n}(t)\right\| \leq\left\|e_{n}(0)\right\|+\left\|F_{n}(t)\right\|+\int_{0}^{t}\|A\|\left\|e_{n}(s)\right\| d s+M \int_{0}^{t}\left\|e_{n}\left(s-\tau_{n}(s)\right)\right\| d s .
$$

Define

$$
X_{n, t}=\left\{s \in[0, t] \mid s-\tau_{n}(s)<0\right\} .
$$

This set is measurable and, since $0 \leq \tau_{n}(t) \leq r_{n}$ for all $t \in \mathbb{R}_{+}$, we have that $X_{n, t} \subset\left[0, r_{n}\right]$, so that $\lambda\left(X_{n, t}\right) \leq r_{n}$ for all $t \in \mathbb{R}_{+}$, where $\lambda$ denotes the Lebesgue measure. Define also

$$
E_{n}(t)=\sup _{s \in\left[t-r_{n}, t\right] \cap[0, t]}\left\|e_{n}(s)\right\|
$$

and $M^{\prime}=\|A\|+M$. From (A.3), we obtain

$$
\left\|e_{n}(t)\right\| \leq\left\|e_{n}(0)\right\|+\left\|F_{n}(t)\right\|+M \int_{X_{n, t}}\left\|e_{n}\left(s-\tau_{n}(s)\right)\right\| d s+M^{\prime} \int_{0}^{t} E_{n}(s) d s,
$$

so that, for $t \geq 0$,

$$
E_{n}(t) \leq \varphi_{n}(t)+M^{\prime} \int_{0}^{t} E_{n}(s) d s
$$


with $\varphi_{n}$ given by $\varphi_{n}(t)=\left\|e_{n}(0)\right\|+\sup _{\sigma \in\left[t-r_{n}, t\right] \cap[0, t]}\left[\left\|F_{n}(\sigma)\right\|+M \int_{X_{n, \sigma}}\left\|e_{n}\left(s-\tau_{n}(s)\right)\right\| d s\right]$. Applying Gronwall's Lemma, we get

$$
E_{n}(t) \leq \varphi_{n}(t)+M^{\prime} \int_{0}^{t} \varphi_{n}(s) e^{M^{\prime}(t-s)} d s
$$

for $t \geq 0$.

Fix $T>0$. Since $\lim _{n \rightarrow+\infty} F_{n}(t)=0$ uniformly on $[0, T]$, we have that

$$
\lim _{n \rightarrow+\infty}\left[\sup _{\sigma \in\left[t-r_{n}, t\right] \cap[0, t]}\left\|F_{n}(\sigma)\right\|\right]=0 \quad \text { uniformly on } t \in[0, T] .
$$

Moreover, for $s \in X_{n, \sigma}$, we have that

$$
\left\|e_{n}\left(s-\tau_{n}(s)\right)\right\|=\left\|x_{n}\left(s-\tau_{n}(s)\right)-x_{\star}\left(s-\tau_{n}(s)\right)\right\| \leq C,
$$

where $C=\Lambda+\sup _{t \in[-r, 0]}\left\|x_{\star}(t)\right\|$, and so

$$
\sup _{\sigma \in\left[t-r_{n}, t\right] \cap[0, t]} \int_{X_{n, \sigma}}\left\|e_{n}\left(s-\tau_{n}(s)\right)\right\| d s \leq C r_{n} \underset{n \rightarrow+\infty}{\longrightarrow} 0
$$

uniformly on $t \in[0, T]$. Hence $\varphi_{n}(t) \underset{n \rightarrow+\infty}{\longrightarrow} 0$ uniformly on $[0, T]$, from where we get, together with (A.4), that $E_{n}(t) \underset{n \rightarrow+\infty}{\longrightarrow} 0$ uniformly on $[0, T]$. So $e_{n}(t) \underset{n \rightarrow+\infty}{\longrightarrow} 0$ uniformly on $[0, T]$, and, since $T>0$ is arbitrary, this gives the desired result.

\section{B Appendix: On the Proof of Theorem 2.5}

We prove here some of the results that were used in the proof of Theorem 2.5. The first three results, Lemmas B.1, B.2 and B.3, deal with the reduction of Theorem 2.5 to the case where $(A, B)$ is controllable, $m=1$ and all the eigenvalues of $A$ lie on the imaginary axis. We begin by reducing the theorem to the case where $(A, B)$ is controllable.

Lemma B.1. It suffices to prove Theorem 2.5 in the case where $(A, B)$ is controllable.

Proof. Up to a linear change of variables, $A$ and $B$ can be decomposed on the controllable and uncontrollable parts according to Kalman decomposition as

$$
A=\left(\begin{array}{cc}
A_{1} & A_{3} \\
0 & A_{2}
\end{array}\right), \quad B=\left(\begin{array}{c}
B_{1} \\
0
\end{array}\right)
$$

with $A_{1} \in \mathcal{M}_{d^{\prime}}(\mathbb{R}), A_{2} \in \mathcal{M}_{d-d^{\prime}}(\mathbb{R}), B_{1} \in \mathcal{M}_{d^{\prime}, m}(\mathbb{R})$, the other matrices having appropriate dimensions, and where $\left(A_{1}, B_{1}\right)$ is controllable (see, for instance, [24, Theorem 13.1]); since $(A, B)$ is stabilizable, $A_{2}$ is Hurwitz. The open-loop system (2.1) can thus be written after the change of variables as

$$
\left\{\begin{array}{l}
\dot{x}_{1}(t)=A_{1} x_{1}(t)+A_{3} x_{2}(t)+\alpha(t) B_{1} u(t), \\
\dot{x}_{2}(t)=A_{2} x_{2}(t)
\end{array}\right.
$$


with $x_{1}(t) \in \mathbb{R}^{d^{\prime}}, x_{2}(t) \in \mathbb{R}^{d-d^{\prime}}$, and $x(t)=\left(x_{1}(t)^{\mathrm{T}} \quad x_{2}(t)^{\mathrm{T}}\right)^{\mathrm{T}}$. Now, suppose the theorem is proved for the controllable case and $K^{\prime} \in \mathcal{M}_{m, d^{\prime}}(\mathbb{R})$ is a $(T, \mu, \mathcal{T})$-stabilizer for $\left(A_{1}, B_{1}\right)$ for a certain neighborhood $\mathcal{T}$ of $\tau_{0}$ in $\mathbb{R}_{+}$, associated with certain constants $C_{1} \geq 1, \gamma_{1}>0$ as in Definition 2.2. Take $K=\left(\begin{array}{ll}K^{\prime} & 0\end{array}\right) \in \mathcal{M}_{m, d}(\mathbb{R})$, so that, with the feedback $u(t)=-K x(t-$ $\tau(t))$, (B.1) becomes

$$
\left\{\begin{array}{l}
\dot{x}_{1}(t)=A_{1} x_{1}(t)-\alpha(t) B_{1} K^{\prime} x_{1}(t-\tau(t))+A_{3} x_{2}(t), \\
\dot{x}_{2}(t)=A_{2} x_{2}(t) .
\end{array}\right.
$$

Let us note $r=\sup \mathcal{T}$. Take $\alpha \in \mathcal{G}(T, \mu), \tau \in L^{\infty}\left(\mathbb{R}_{+}, \mathcal{T}\right)$, and an initial condition $x_{0} \in$ $\mathrm{C}^{0}\left([-r, 0], \mathbb{R}^{d}\right)$, written as $x_{0}(t)=\left(x_{0,1}(t)^{\mathrm{T}} \quad x_{0,2}(t)^{\mathrm{T}}\right)^{\mathrm{T}}$. Note by $y(t) \in \mathbb{R}^{d}$ the solution of

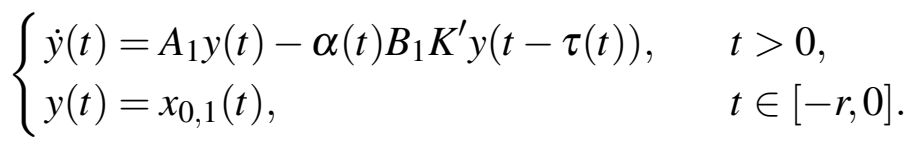

Then, by the hypothesis on $K^{\prime}$, we have that

$$
\|y(t)\| \leq C_{1} e^{-\gamma_{1} t} \sup _{s \in[-r, 0]}\left\|x_{0,1}(s)\right\| .
$$

The result on [12, Section 6.2] allows us to write the solution $x(t)=\left(x_{1}(t)^{\mathrm{T}} \quad x_{2}(t)^{\mathrm{T}}\right)^{\mathrm{T}}$ of (B.2) associated with $\alpha$ and $\tau$ and with initial condition $x_{0}$ as

$$
\left\{\begin{array}{l}
x_{1}(t)=y(t)+\int_{0}^{t} X(t, s) A_{3} x_{2}(s) d s, \\
x_{2}(t)=e^{A_{2} t} x_{0,2}(0)
\end{array}\right.
$$

where $X(t, s) \in \mathcal{M}_{d^{\prime}}(\mathbb{R})$ is the fundamental matrix solution associated with the delayed system $\dot{z}(t)=A_{1} z(t)-\alpha(t) B_{1} K^{\prime} z(t-\tau(t))$ (see [12, Section 6.1]). Our choice of $K^{\prime}$ guarantees that this last system is exponentially stable, uniformly with respect to $\alpha \in \mathcal{G}(T, \mu)$ and $\tau \in L^{\infty}\left(\mathbb{R}_{+}, \mathcal{T}\right)$, and so, by [12, Lemma 6.5.3], there exist constants $C_{0} \geq 1, \gamma_{0}>0$ independent of $\alpha$ and $\tau$ such that

$$
\|X(t, s)\| \leq C_{0} e^{-\gamma_{0}(t-s)} \quad \text { for all } t \geq s \geq 0 ;
$$

note that [12, Lemma 6.5.3] is proved only for the case of uniformity with respect to the initial time, but the same proof also applies for the case of uniformity with respect to other parameters. Note also that we do not need to consider uniformity with respect to the initial time since the classes $\mathcal{G}(T, \mu)$ and $L^{\infty}\left(\mathbb{R}_{+}, \mathcal{T}\right)$ are invariant with respect to positive time translations and a non-zero initial time may be translated into terms of a different choice of $\alpha$ and $\tau$.

Since $A_{2}$ is Hurwitz, there exist $C_{2} \geq 1, \gamma_{2}>0$ such that

$$
\left\|e^{A_{2} t}\right\| \leq C_{2} e^{-\gamma_{2} t} .
$$

Using the estimates (B.3), (B.5) and (B.6) in (B.4), we can find $C \geq 1$ and $\gamma>0$, depending only on $C_{0}, C_{1}, C_{2}, \gamma_{0}, \gamma_{1}, \gamma_{2}$, and thus independent of $\alpha$ and $\tau$, such that

$$
\|x(t)\| \leq C e^{-\gamma t} \sup _{s \in[-r, 0]}\left\|x_{0}(s)\right\|
$$

which proves that $K$ is a $(T, \mu, \mathcal{T})$-stabilizer for $(A, B)$, as desired. 
The following lemma shows that we may further reduce Theorem 2.5 to the single-input case. Its proof follows the same idea of [9, Chapter 4, Theorem 4], where the original stabilization result for single-input systems of [10, Theorem 3.2] is generalized to the multi-input case by a recurrence on the number of inputs.

Lemma B.2. It suffices to prove Theorem 2.5 in the case where $(A, B)$ is controllable and $m=1$.

Proof. We may suppose $(A, B)$ controllable by Lemma B.1. We suppose the theorem to be proved in the case $m=1$ and we prove the general case by induction on $m$. Suppose the theorem has been proved for $m-1$, that is, for every $d \in \mathbb{N}^{*}$, for every $A \in \mathcal{M}_{d}(\mathbb{R})$ and $B \in \mathcal{M}_{d, m-1}(\mathbb{R})$ such that $(A, B)$ is a controllable pair and the eigenvalues of $A$ have non-positive real part, for every $T, \mu$ with $T \geq \mu>0$, and for every $\tau_{0} \geq 0$, there exists a neighborhood $\mathcal{T}$ of $\tau_{0}$ in $\mathbb{R}_{+}$and a $(T, \mu, \mathcal{T})$-stabilizer for (2.2).

Take $A \in \mathcal{M}_{d}(\mathbb{R})$ and $B \in \mathcal{M}_{d, m}(\mathbb{R})$ such that $(A, B)$ is a controllable pair and the eigenvalues of $A$ have non-positive real part and fix $T \geq \mu>0$ and $\tau_{0} \geq 0$. Note by $b \in \mathbb{R}^{d}$ the first column of $B$; we may suppose, without loss of generality, that $b \neq 0$, for otherwise the first input does not influence the system and it may thus be excluded, reducing the system to the case with $m-1$ inputs. We consider the pair $(A, b)$, which may not be controllable, but can be decomposed according to Kalman decomposition: there exists an invertible $P \in \mathcal{M}_{d}(\mathbb{R})$ such that

$$
P A P^{-1}=\left(\begin{array}{cc}
A_{1} & A_{3} \\
0 & A_{2}
\end{array}\right), \quad P b=\left(\begin{array}{c}
b_{1} \\
0
\end{array}\right),
$$

with $A_{1} \in \mathcal{M}_{d^{\prime}}(\mathbb{R}), b_{1} \in \mathbb{R}^{d^{\prime}}$, all the other matrices have appropriate dimensions, and $\left(A_{1}, b_{1}\right)$ is controllable. Now, performing the change of variables $z=P x$ in (2.1), the open-loop system becomes

$$
\dot{z}=\left(\begin{array}{cc}
A_{1} & A_{3} \\
0 & A_{2}
\end{array}\right) z+\alpha(t)\left(\begin{array}{cc}
b_{1} & B_{3} \\
0 & B_{2}
\end{array}\right) u
$$

with $B_{2} \in \mathcal{M}_{d-d^{\prime}, m-1}(\mathbb{R})$ and $B_{3} \in \mathcal{M}_{d^{\prime}, m-1}(\mathbb{R})$.

By the controllability of $(A, B)$ and $\left(A_{1}, b_{1}\right)$, it follows that $\left(A_{2}, B_{2}\right)$ is also controllable. Now $B_{2} \in \mathcal{M}_{d-d^{\prime}, m-1}(\mathbb{R})$, and so, by the induction hypothesis, $\left(A_{2}, B_{2}\right)$ admits a $\left(T, \mu, \mathcal{T}_{2}\right)$ stabilizer $K_{2} \in \mathcal{M}_{m-1, d-d^{\prime}}(\mathbb{R})$ for a certain neighborhood $\mathcal{T}_{2}$ of $\tau_{0}$ in $\mathbb{R}_{+}$. If Theorem 2.5 is proved in the controllable case with $m=1$, then we can take a $\left(T, \mu, \mathcal{T}_{1}\right)$-stabilizer $K_{1} \in$ $\mathcal{M}_{1, d^{\prime}}(\mathbb{R})$ for $\left(A_{1}, b_{1}\right)$ for a certain neighborhood $\mathcal{T}_{1}$ of $\tau_{0}$ in $\mathbb{R}_{+}$. We claim that $K \in \mathcal{M}_{m, d}(\mathbb{R})$ given by

$$
K=\left(\begin{array}{cc}
K_{1} & 0 \\
0 & K_{2}
\end{array}\right)
$$

is a $(T, \mu, \mathcal{T})$-stabilizer for $(A, B)$ for the neighborhood $\mathcal{T}=\mathcal{T}_{1} \cap \mathcal{T}_{2}$. Indeed, with this feedback, system (B.7) becomes

$$
\dot{z}(t)=\left(\begin{array}{cc}
A_{1} & A_{3} \\
0 & A_{2}
\end{array}\right) z(t)-\alpha(t)\left(\begin{array}{cc}
b_{1} K_{1} & B_{3} K_{2} \\
0 & B_{2} K_{2}
\end{array}\right) z(t-\tau(t)) .
$$

Noting $z=\left(\begin{array}{ll}z_{1}^{\mathrm{T}} & z_{2}^{\mathrm{T}}\end{array}\right)^{\mathrm{T}}$ with $z_{1} \in \mathbb{R}^{d^{\prime}}$ and $z_{2} \in \mathbb{R}^{d-d^{\prime}}$, we can thus write

$$
\left\{\begin{array}{l}
\dot{z}_{1}(t)=A_{1} z_{1}(t)-\alpha(t) b_{1} K_{1} z_{1}(t-\tau(t))+A_{3} z_{2}(t)-\alpha(t) B_{3} K_{2} z_{2}(t-\tau(t)), \\
\dot{z}_{2}(t)=A_{2} z_{2}(t)-\alpha(t) B_{2} K_{2} z_{2}(t-\tau(t)) .
\end{array}\right.
$$


We denote by $X(t, s)$ the fundamental matrix solution of $\dot{x}(t)=A_{1} x(t)-\alpha(t) b_{1} K_{1} x(t-$ $\tau(t))$; by construction of $K_{1}$ and by [12, Lemma 6.5.3], we can find $C_{0} \geq 1$ and $\gamma_{0}>0$, both independent of $\alpha \in \mathcal{G}(T, \mu)$ and $\tau \in L^{\infty}\left(\mathbb{R}_{+}, \mathcal{T}\right)$, such that

$$
\|X(t, s)\| \leq C_{0} e^{-\gamma_{0}(t-s)}, \quad \forall t \geq s \geq 0 .
$$

Note $r=\sup \mathcal{T}$. Given an initial condition $\left(z_{0,1}^{\mathrm{T}} \quad z_{0,2}^{\mathrm{T}}\right)^{\mathrm{T}} \in \mathcal{C}^{0}\left([-r, 0], \mathbb{R}^{d}\right)$, note by $y_{1}$ and $y_{2}$ the solutions to

$$
\begin{array}{ll}
\dot{y}_{1}(t)=A_{1} y_{1}(t)-\alpha(t) b_{1} K_{1} y_{1}(t-\tau(t)), & y_{1}(t)=z_{0,1}(t) \text { for } t \in[-r, 0], \\
\dot{y}_{2}(t)=A_{2} y_{2}(t)-\alpha(t) B_{2} K_{2} y_{2}(t-\tau(t)), & y_{2}(t)=z_{0,2}(t) \text { for } t \in[-r, 0] .
\end{array}
$$

By construction of $K_{1}$ and $K_{2}$, there exist $C_{1}, C_{2} \geq 1$ and $\gamma_{1}, \gamma_{2}>0$ such that

$$
\left\|y_{j}(t)\right\| \leq C_{j} e^{-\gamma_{j} t} \sup _{s \in[-r, 0]}\left\|z_{0, j}(s)\right\|, \quad j=1,2 .
$$

We can now write the solution of (B.8) in terms of the initial condition $\left(z_{0,1}^{\mathrm{T}} \quad z_{0,2}^{\mathrm{T}}\right)^{\mathrm{T}} \in$ $\mathrm{C}^{0}\left([-r, 0], \mathbb{R}^{d}\right)$ using the variation-of-constants formula in [12, Section 6.2] as

$$
\left\{\begin{array}{l}
z_{1}(t)=y_{1}(t)+\int_{0}^{t} X(t, s)\left(A_{3} z_{2}(s)-\alpha(s) B_{3} K_{2} z_{2}(s-\tau(s))\right) d s \\
z_{2}(t)=y_{2}(t) .
\end{array}\right.
$$

It is thus easy to see that

$$
\left\{\begin{array}{l}
\left\|z_{1}(t)\right\| \leq C_{1} e^{-\gamma_{1} t} \sup _{s \in[-r, 0]}\left\|z_{0,1}(s)\right\|+C^{\prime} e^{-\gamma^{\prime} t} \sup _{s \in[-r, 0]}\left\|z_{0,2}(s)\right\|, \\
\left\|z_{2}(t)\right\| \leq C_{2} e^{-\gamma_{2} t} \sup _{s \in[-r, 0]}\left\|z_{0,2}(s)\right\|
\end{array}\right.
$$

for certain constants $C^{\prime} \geq 1, \gamma^{\prime}>0$, and so $K$ is a $(T, \mu, \mathcal{T})$-stabilizer for (B.7), as we wanted to prove. The result is thus established by induction.

We further reduce our proof of Theorem 2.5 to the case where all the eigenvalues of $A$ lie on the imaginary axis.

Lemma B.3. It suffices to prove Theorem 2.5 in the case where $(A, B)$ is controllable, $m=1$, and $\Re(\lambda)=0$ for every eigenvalue $\lambda$ of $A$.

Proof. We may suppose $(A, B)$ controllable and $m=1$ by Lemma B.2. Up to a linear change of variables, $A$ and $B$ can be written as

$$
A=\left(\begin{array}{cc}
A_{1} & A_{3} \\
0 & A_{2}
\end{array}\right), \quad B=\left(\begin{array}{l}
B_{1} \\
B_{2}
\end{array}\right)
$$

with $A_{1} \in \mathcal{M}_{d^{\prime}}(\mathbb{R}), A_{2} \in \mathcal{M}_{d-d^{\prime}}(\mathbb{R}), B_{1} \in \mathbb{R}^{d^{\prime}}$, the other matrices having appropriate dimensions, and where $A_{1}$ is Hurwitz and all the eigenvalues of $A_{2}$ have real part 0 . Since $(A, B)$ is controllable, $\left(A_{2}, B_{2}\right)$ is also controllable. The open-loop system (2.1) can thus be written after the change of variables as

$$
\left\{\begin{array}{l}
\dot{x}_{1}(t)=A_{1} x_{1}(t)+A_{3} x_{2}(t)+\alpha(t) B_{1} u(t) \\
\dot{x}_{2}(t)=A_{2} x_{2}(t)+\alpha(t) B_{2} u(t)
\end{array}\right.
$$


with $x_{1}(t) \in \mathbb{R}^{d^{\prime}}, x_{2}(t) \in \mathbb{R}^{d-d^{\prime}}$, and $x(t)=\left(x_{1}(t)^{\mathrm{T}} \quad x_{2}(t)^{\mathrm{T}}\right)^{\mathrm{T}}$. Now, suppose the theorem is proved for the case stated above and take $K^{\prime} \in \mathcal{M}_{1, d-d^{\prime}}(\mathbb{R})$ a $(T, \mu, \mathcal{T})$-stabilizer for $\left(A_{2}, B_{2}\right)$ for a certain neighborhood $\mathcal{T}$ of $\tau_{0}$ in $\mathbb{R}_{+}$, associated with certain constants $C_{2} \geq 1, \gamma_{2}>0$ as in Definition 2.2. Take $K=\left(\begin{array}{ll}0 & K^{\prime}\end{array}\right) \in \mathcal{M}_{1, d}(\mathbb{R})$, so that, with the feedback $u(t)=-K x(t-$ $\tau(t)),($ B.10) becomes

$$
\left\{\begin{array}{l}
\dot{x}_{1}(t)=A_{1} x_{1}(t)+A_{3} x_{2}(t)-\alpha(t) B_{1} K^{\prime} x_{2}(t-\tau(t)), \\
\dot{x}_{2}(t)=A_{2} x_{2}(t)-\alpha(t) B_{2} K^{\prime} x_{2}(t-\tau(t))
\end{array}\right.
$$

Let us note $r=\sup \mathcal{T}$. Take $\alpha \in \mathcal{G}(T, \mu), \tau \in L^{\infty}\left(\mathbb{R}_{+}, \mathcal{T}\right)$, and an initial condition $x_{0} \in$ $\mathcal{C}^{0}\left([-r, 0], \mathbb{R}^{d}\right)$, written as $x_{0}(t)=\left(x_{0,1}(t)^{\mathrm{T}} \quad x_{0,2}(t)^{\mathrm{T}}\right)^{\mathrm{T}}$. By the hypothesis on $K^{\prime}$, we have that the solution $x(t)=\left(x_{1}(t)^{\mathrm{T}} \quad x_{2}(t)^{\mathrm{T}}\right)^{\mathrm{T}}$ of (B.11) associated with $\alpha$ and $\tau$ and with initial condition $x_{0}$ satisfies

$$
\left\|x_{2}(t)\right\| \leq C_{2} e^{-\gamma_{2} t} \sup _{s \in[-r, 0]}\left\|x_{2}(s)\right\| .
$$

Applying the variation-of-constants formula to (B.11) and using an exponential estimate on $\left\|e^{A_{1} t}\right\|$, it is immediate to verify that $K$ is a $(T, \mu, \mathcal{T})$-stabilizer for $(A, B)$.

Let us now present a proof of Lemma 4.1, which was originally done in [10] and that we recall here for the sake of completeness.

Proof of Lemma 4.1. Up to a linear change of variables in (2.1), we may suppose that $A$ is in its real Jordan normal form. $A$ has a unique Jordan block associated with each $\left\{-i \omega_{j}, i \omega_{j}\right\}, j=j_{0}, \ldots, h$, for, otherwise, the rank of the matrix $\left(\begin{array}{ll}A-i \omega_{j} \operatorname{Id}_{d} & B\end{array}\right)$ would be strictly smaller than $d$, contradicting the Hautus test for controllability. Thus, up to a permutation of variables on $\mathbb{R}^{d}$, we can write $A=\operatorname{diag}\left(J_{r_{0}}, \omega_{1} A^{(1)}+J_{r_{1}}^{C}, \ldots, \omega_{h} A^{(h)}+J_{r_{h}}^{C}\right)$, and $B \in$

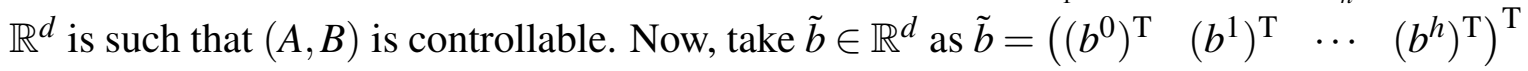
with $b^{0}$ and $b^{j}, j=1, \ldots, h$, as defined in the statement of the lemma. It follows from Hautus test for controllability that $(A, \tilde{b})$ is controllable. But all controllable linear control systems associated with a pair $(A, B)$ that have in common the eigenvalues of $A$, counted according to their multiplicity, are state-equivalent, since they can be transformed by a linear transformation of coordinates into the same system under controller form (see, e.g., [26]), and so $(A, B)$ can be transformed into $(A, \tilde{b})$ by a linear transformation of coordinates, leading to the desired result.

Finally, to complete the proof of Theorem 2.5, we prove Lemma 4.2, which gives the uniform exponential stability of the limit system considered in the proof of Theorem 2.5.

Proof of Lemma 4.2. We consider the matrices $P_{j \ell}$ as a perturbations in (4.7), and so we consider first the non-perturbed system

$$
\left\{\begin{array}{l}
\dot{y}_{0}(t)=J_{r_{0}} y_{0}(t)-\sum_{\ell=0}^{h}\left[b^{0} \mathcal{K}_{\ell} \otimes C_{0 \ell}(t)\right] y_{\ell}(t), \\
\dot{y}_{j}(t)=J_{r_{j}}^{C} y_{j}(t)-\sum_{\ell=0}^{h}\left[\tilde{b}^{j} \mathcal{K}_{\ell} \otimes C_{j \ell}(t)\right] y_{\ell}(t), \quad j=1, \ldots, h .
\end{array}\right.
$$

Let $\xi>0$. It has been proved in [10, Theorem 3.2] that, for a given $\xi>0$, one can find a gain $\mathcal{K}=\left(\begin{array}{llll}\mathcal{K}_{0} & \mathcal{K}_{1} & \cdots & \mathcal{K}_{h}\end{array}\right)$ and a positive definite matrix $S \in \mathcal{M}_{d}(\mathbb{R})$ such that, for every symmetric $C_{\star} \in L^{\infty}\left(\mathbb{R}_{+}, \mathcal{M}{ }_{2 h+1-j_{0}}(\mathbb{R})\right)$ satisfying $C_{\star}(t) \geq \xi \mathrm{Id}_{2 h+1-j_{0}}$ for almost every 
$t \geq 0$, (B.12) is globally uniformly exponentially stable and $V(y)=y^{\mathrm{T}} S y$ decreases exponentially along all trajectories of (B.12), uniformly with respect to $C_{\star} \in L^{\infty}\left(\mathbb{R}_{+}, \mathcal{M}_{2 h+1-j_{0}}(\mathbb{R})\right)$ satisfying $C_{\star}(t) \geq \xi \mathrm{Id}_{2 h+1-j_{0}}$ almost everywhere; i.e., there exist $C \geq 1$ and $\gamma>0$ such that, for every symmetric $C_{\star} \in L^{\infty}\left(\mathbb{R}_{+}, \mathcal{M}_{2 h+1-j_{0}}(\mathbb{R})\right)$ satisfying $C_{\star}(t) \geq \xi \operatorname{Id}_{2 h+1-j_{0}}$ almost everywhere and every solution $y$ of (B.12), we have

$$
\|y(t)\| \leq C e^{-2 \gamma t}\|y(0)\| .
$$

We denote by $X(t, s)$ the fundamental matrix solution of (B.12), i.e., for any $y^{0} \in \mathbb{R}^{d}, y(t)=$ $X(t, s) y^{0}$ is the unique solution to (B.12) with $y(s)=y^{0}$. Hence we have the estimate

$$
\|X(t, s)\| \leq C e^{-2 \gamma(t-s)} .
$$

We now turn to the perturbed system (4.7). For a given $\xi>0$, we take $C \geq 1, \gamma>0$ and $\mathcal{K}_{j}$ as before. For every symmetric matrix $C_{\star} \in L^{\infty}\left(\mathbb{R}_{+}, \mathcal{M}{ }_{2 h+1-j_{0}}(\mathbb{R})\right)$ satisfying $C_{\star}(t) \geq$ $\xi \operatorname{Id}_{2 h+1-j_{0}}$ almost everywhere, and every $P_{\star} \in L^{\infty}\left(\mathbb{R}_{+}, \mathcal{M}_{2 h+1-j_{0}}(\mathbb{R})\right)$ satisfying (4.9), we set $\mathcal{A}=\operatorname{diag}\left(J_{r_{0}}, J_{r_{1}}^{C}, \ldots, J_{r_{h}}^{C}\right) \in \mathcal{M}_{d}(\mathbb{R})$,

$$
\mathcal{B}(t)=\left(\tilde{b}^{j} \mathcal{K}_{\ell} \otimes C_{j \ell}(t)\right)_{j_{0} \leq j, \ell \leq h}, \quad \mathcal{P}(t)=\left(\tilde{b}^{j} \mathcal{K}_{\ell} \otimes P_{j \ell}(t)\right)_{j_{0} \leq j, \ell \leq h}
$$

with $\tilde{b}^{0}=b^{0}$. System (4.7) can thus be written under the form

$$
\dot{y}(t)=\mathcal{A} y(t)-\mathcal{B}(t) y(t)-\mathcal{P}(t) y(t)
$$

and, using the fundamental matrix $X$ of (B.12), we can write its solution for a given initial condition $y^{0}$ as

$$
y(t)=X(t, 0) y^{0}-\int_{0}^{t} X(t, s) \mathcal{P}(s) y(s) d s .
$$

By (4.9), we can write $\|\mathcal{P}(t)\| \leq C^{\prime} r \Omega$ for a certain constant $C^{\prime}>0$, and thus, up to increasing $C$, we have, by (B.13),

$$
\|y(t)\| \leq C e^{-2 \gamma t}\left\|y^{0}\right\|+C r \Omega \int_{0}^{t} e^{-2 \gamma(t-s)}\|y(s)\| d s .
$$

Applying Gronwall's Lemma to $e^{2 \gamma t}\|y(t)\|$, we thus obtain

$$
\|y(t)\| \leq C e^{-(2 \gamma-C r \Omega) t}\left\|y^{0}\right\| .
$$

We choose $r>0$ small enough so that $2 \gamma-C r \Omega \geq \gamma$, and so

$$
\|y(t)\| \leq C e^{-\gamma t}\left\|y^{0}\right\|
$$

which gives us the desired result.

\section{Acknowledgment}

The author would like to thank Y. Chitour and M. Sigalotti for having suggested the study of this problem and for the helpful discussions that followed. 


\section{References}

[1] B. Anderson. Exponential stability of linear equations arising in adaptive identification. IEEE Trans. Automat. Control, 22(1):83-88, 1977.

[2] B. Anderson, R. Bitmead, C. Johnson, P. Kokotovic, R. Kosut, I. Mareels, L. Praly, and B. Riedle. Stability of adaptive systems: Passivity and averaging analysis. MIT Press Series in Signal Processing, Optimization, and Control, 8. MIT Press, Cambridge, MA, 1986.

[3] S. Andersson and P. Krishnaprasad. Degenerate gradient flows: a comparison study of convergence rate estimates. In Decision and Control, 2002, Proceedings of the 41st IEEE Conference on, volume 4, pages 4712-4717. IEEE, 2002.

[4] M. Balde, U. Boscain, and P. Mason. A note on stability conditions for planar switched systems. Internat. J. Control, 82(10):1882-1888, 2009.

[5] U. Boscain. Stability of planar switched systems: the linear single input case. SIAM J. Control Optim., 41(1):89-112, 2002.

[6] R. Brockett. The rate of descent for degenerate gradient flows. In Proceedings of the 2000 MTNS, 2000.

[7] A. Chaillet, Y. Chitour, A. Loría, and M. Sigalotti. Uniform stabilization for linear systems with persistency of excitation: the neutrally stable and the double integrator cases. Math. Control Signals Systems, 20(2):135-156, 2008.

[8] A. Chaillet, Y. Chitour, A. Loria, and M. Sigalotti. Towards uniform linear time-invariant stabilization of systems with persistency of excitation. In Decision and Control, 2007 46th IEEE Conference on, pages 6394-6399. IEEE, 2007.

[9] Y. Chitour, G. Mazanti, and M. Sigalotti. Stabilization of persistently excited linear systems. In J. Daafouz, S. Tarbouriech, and M. Sigalotti, editors, Hybrid Systems with Constraints, chapter 4. Wiley-ISTE, London, UK, 2013.

[10] Y. Chitour and M. Sigalotti. On the stabilization of persistently excited linear systems. SIAM J. Control Optim., 48(6):4032-4055, 2010.

[11] J.-P. Gauthier and I. A. K. Kupka. Observability and observers for nonlinear systems. SIAM J. Control Optim., 32(4):975-994, 1994.

[12] J. K. Hale and S. M. Verduyn Lunel. Introduction to functional differential equations, volume 99 of Applied Mathematical Sciences. Springer-Verlag, New York, 1993.

[13] F. Hante, M. Sigalotti, and M. Tucsnak. On conditions for asymptotic stability of dissipative infinite-dimensional systems with intermittent damping. Journal of Differential Equations, 252(10):5569-5593, 2012.

[14] D. Liberzon. Switching in Systems and Control. Birkhäuser Boston, 1 edition, 2003.

[15] H. Lin and P. J. Antsaklis. Stability and stabilizability of switched linear systems: a survey of recent results. IEEE Trans. Automat. Control, 54(2):308-322, 2009. 
[16] A. Loria, A. Chaillet, G. Besançon, and Y. Chitour. On the PE stabilization of timevarying systems: open questions and preliminary answers. In Decision and Control, 2005 and 2005 European Control Conference. CDC-ECC'05. 44th IEEE Conference on, pages 6847-6852. IEEE, 2005.

[17] M. Lovera and A. Astolfi. Global spacecraft attitude control using magnetic actuators. In Advances in dynamics and control, volume 2 of Nonlinear Syst. Aviat. Aerosp. Aeronaut. Astronaut., pages 1-13. Chapman \& Hall/CRC, Boca Raton, FL, 2004.

[18] M. Malisoff and F. Mazenc. Constructions of strict Lyapunov functions. Communications and Control Engineering Series. Springer-Verlag London Ltd., London, 2009.

[19] M. Margaliot. Stability analysis of switched systems using variational principles: an introduction. Automatica, 42(12):2059-2077, 2006.

[20] G. Mazanti, Y. Chitour, and M. Sigalotti. Stabilization of two-dimensional persistently excited linear control systems with arbitrary rate of convergence. SIAM J. Control Optim., 51(2):801-823, 2013.

[21] W. Michiels and S.-I. Niculescu. Stability and stabilization of time-delay systems: An eigenvalue-based approach, volume 12 of Advances in Design and Control. Society for Industrial and Applied Mathematics (SIAM), Philadelphia, PA, 2007.

[22] A. P. Morgan and K. S. Narendra. On the stability of nonautonomous differential equations $\dot{x}=[A+B(t)] x$ with skew-symmetric matrix $B(t)$. SIAM J. Control Optim., 15(1):163-176, 1977.

[23] S.-I. Niculescu. Delay effects on stability: A robust control approach, volume 269 of Lecture Notes in Control and Information Sciences. Springer-Verlag London Ltd., London, 2001.

[24] W. J. Rugh. Linear System Theory. Prentice Hall, 2 edition, 1996.

[25] R. Shorten, F. Wirth, O. Mason, K. Wulff, and C. King. Stability criteria for switched and hybrid systems. SIAM Rev., 49(4):545-592, 2007.

[26] E. D. Sontag. Mathematical control theory, volume 6 of Texts in Applied Mathematics. Springer-Verlag, New York, 2 edition, 1998. Deterministic finite-dimensional systems. 